\title{
Arsenic in drinking water and urinary tract cancers: a systematic review of 30 years of epidemiological evidence
}

\author{
Nathalie Saint-Jacques ${ }^{1,2^{*}}$, Louise Parker ${ }^{3}$, Patrick Brown ${ }^{4}$ and Trevor JB Dummer ${ }^{3}$
}

\begin{abstract}
Background: Arsenic in drinking water is a public health issue affecting hundreds of millions of people worldwide. This review summarizes 30 years of epidemiological studies on arsenic exposure in drinking water and the risk of bladder or kidney cancer, quantifying these risks using a meta-analytical framework.

Methods: Forty studies met the selection criteria. Seventeen provided point estimates of arsenic concentrations in drinking water and were used in a meta-analysis of bladder cancer incidence (7 studies) and mortality (10 studies) and kidney cancer mortality (2 studies). Risk estimates for incidence and mortality were analyzed separately using Generalized Linear Models. Predicted risks for bladder cancer incidence were estimated at 10,50 and $150 \mathrm{\mu g} / \mathrm{L}$ arsenic in drinking water. Bootstrap randomizations were used to assess robustness of effect size.

Results: Twenty-eight studies observed an association between arsenic in drinking water and bladder cancer. Ten studies showed an association with kidney cancer, although of lower magnitude than that for bladder cancer. The meta-analyses showed the predicted risks for bladder cancer incidence were 2.7 [1.2-4.1]; 4.2 [2.1-6.3] and; 5.8 [2.9-8.7] for drinking water arsenic levels of 10,50 , and $150 \mu \mathrm{g} / \mathrm{L}$, respectively. Bootstrapped randomizations confirmed this increased risk, but, lowering the effect size to 1.4 [0.35-4.0], 2.3 [0.59-6.4], and 3.1 [0.80-8.9]. The latter suggests that with exposures to $50 \mu \mathrm{g} / \mathrm{L}$, there was an $83 \%$ probability for elevated incidence of bladder cancer; and a 74\% probability for elevated mortality. For both bladder and kidney cancers, mortality rates at $150 \mathrm{ug} / \mathrm{L}$ were about 30\% greater than those at $10 \mu \mathrm{g} / \mathrm{L}$.

Conclusion: Arsenic in drinking water is associated with an increased risk of bladder and kidney cancers, although at lower levels $(<150 \mu \mathrm{g} / \mathrm{L})$, there is uncertainty due to the increased likelihood of exposure misclassification at the lower end of the exposure curve. Meta-analyses suggest exposure to $10 \mu \mathrm{g} / \mathrm{L}$ of arsenic in drinking water may double the risk of bladder cancer, or at the very least, increase it by about $40 \%$. With the large number of people exposed to these arsenic concentrations worldwide the public health consequences of arsenic in drinking water are substantial.
\end{abstract}

Keywords: Arsenic, Drinking water, Bladder, Kidney, Urinary tract, Cancer risk, Systematic review, Meta-analysis

\footnotetext{
* Correspondence: nathalie.st-jacques@ccns.nshealth.ca

${ }^{1}$ Cancer Care Nova Scotia, Surveillance and Epidemiology Unit, Room 560

Bethune Building, 1276 South Street, Halifax B3H 2Y9, Nova Scotia, Canada

2Interdisciplinary PhD program, Dalhousie University, 6299 South Street,

Room 314, PO Box 15000, Halifax B3H 4R2, Nova Scotia, Canada

Full list of author information is available at the end of the article
} 


\section{Background}

Arsenic (As) is a naturally occurring toxic metalloid prevalent in the earth's crust [1]. It enters drinking-water sources in a dissolved state primarily resulting from the weathering of rocks [2]. Human exposure to As involve multiple pathways [3-9], with drinking water being the primary route of exposure for the majority of highly exposed populations $[4,9,10]$. West Bengal, Bangladesh and Taiwan are the most affected regions worldwide [4,11-14]. In these areas, As concentration as high as $4,700 \mu \mathrm{g} / \mathrm{L}$ have been reported in drinking water, and levels in excess of $300 \mu \mathrm{g} / \mathrm{L}$ are common. High levels of As in drinking water have also been reported elsewhere, such as North and South America, Central and Eastern Europe as well as Australia [4,11,15-22].

The contamination of drinking water by As has become an ongoing public health issue affecting hundreds of millions of people worldwide. A growing body of evidence supporting a wide range of acute and chronic effects on health, including cancer [5,20-72], has led the World Health Organization (WHO) to lower the advisory limit for concentration of As in drinking water from $25 \mu \mathrm{g} / \mathrm{L}$ to a provisional guideline limit of $10 \mu \mathrm{g} / \mathrm{L}$ [10]. However, many developing countries continue to endorse an effective upper limit of $50 \mu \mathrm{g} / \mathrm{L}$ [4].

The International Agency for Research on Cancer (IARC) has classified inorganic As in drinking water as a Group 1 carcinogen [73]. Suggested mechanisms of action for As carcinogenesis include oxidative damage, epigenetic effects and interference with DNA repair, mechanisms which have been specifically implicated in the development of As-related urinary tract cancers which are the focus of this review [74-81]. Urinary tract cancers comprise primarily cancers of the urinary bladder and kidney, the former being the ninth most common cause of cancer worldwide [82]. Most studies generally report on bladder or kidney cancer, although some of the studies included in this review and meta-analysis reported histologies, mostly urothelial/transitional cell and renal cell carcinomas. Tobacco smoking and most notably, the ingestion of high levels of inorganic As are two important risk factors for bladder and kidney cancers [83-86].

To date, epidemiological studies of populations exposed to high levels of inorganic As have shown strong associations and dose-response relationships between As in drinking water and bladder cancer and; potential associations with kidney cancer [23]. Typically, these studies report on areas of extreme exposure where levels of As in drinking water range from 150 to over $1000 \mathrm{ug} / \mathrm{L}$. The extent to which health effects may develop remain uncertain at lower levels of exposure $(<150 \mu \mathrm{g} / \mathrm{L})$, with many studies failing to demonstrate the risk that might be expected by extrapolation from findings related to high levels of exposure [5].
This paper reviews findings from epidemiological studies published over the past 30 years, including a number of recent publications focusing on low-levels exposure and bladder and kidney cancer outcomes $[60,63,67,87]$. It also quantifies the risk of urinary tract cancers due to exposure to As in drinking water, combining risk estimates from published epidemiological data. As such, this work complements the recent systematic review of IARC which reports on carcinogenicity following exposure to As [23].

Most studies reporting on urinary cancers risk and As exposure tend to focus on specific levels of exposure. By combining exposure levels from multiple studies, the review profiles a more complete and continuous range of As exposure from which to better assess and predict cancer risks associated with varying levels of exposure. This meta-analytical approach is especially relevant to shed light on dose-response relationship, especially at the lower end of the curve where there has been the most uncertainty and where a large number of people may be at risk.

\section{Methodology}

\section{Review process}

Searches of the Medline (PubMed) and Embase databases were conducted to identify studies reporting on exposure to As in drinking water and urinary tract cancer outcomes and published prior to January 2013. The search conditions are presented in Table 1. Searches were also undertaken using Google Scholar and the WHO and the IARC publications $[3,23]$. Studies were selected based on the selection criteria listed in Table 1. Information abstracted from reviewed articles is shown in Tables 2, 3, 4, 5, 6 . When the distribution of As in drinking water was detailed in another publication, that information was also retrieved. Where available, the adjusted relative risks estimates and associated 95\% confidence intervals were selected.

\section{Data analysis}

Epidemiologic data from studies which explicitly provided point estimates of As levels in drinking water were used in a meta-analysis to examine the association between cancer outcomes and As exposure over a broader and more continuous range of As than previously available (Tables 2, 3, 4, 5, 6, studies with an asterisk). Studies using cumulative exposure to As in drinking water, years of artesian well water consumption or As toenail/urine concentrations were not included in the meta-analyses. Risk estimates from studies reporting on bladder cancer mortality (10 studies) were analysed separately from those reporting on incidence (7 studies). With regards to kidney cancer, only risk estimates for mortality could be analysed 
Table 1 Search conditions and criteria for study selection

\begin{tabular}{l}
\hline Search conditions \\
\hline ((arsenic) AND ("bladder cancer*" OR "kidney cancer*" OR "urinary tract cancer*" OR \\
"upper urinary tract cancer*" OR "urinary tract cancer*" OR "urologic neoplasm*" OR \\
"cancer*, urinary tract" OR "kidney neoplasm*" OR "carcinoma, renal cell" OR "urinary \\
bladder neoplasm*" OR "urinary tract disease*" OR "kidney tumour*" OR "bladder $^{*}$ tumour*" OR "bladder tumor*"OR "kidney tumor*" OR renal cell* carcinoma" OR \\
"bladder neoplasms") AND ("water" OR "drinking water" OR "water supply" OR \\
"toenail" OR "urine" OR "well water") ${ }^{+}$
\end{tabular}

"toenail OR "urine" OR "well water") ${ }^{\dagger}$

\author{
Study selection \\ 1. Arsenic in drinking water, toenail or urine, as exposure \\ of primary interest. \\ 2. Urinary tract cancers incidence and mortality as primary \\ outcome. \\ 3. Original study that published the data. \\ 4. Relative risk estimates, measures of variability (i.e., \\ confidence intervals) documented. \\ 5. Epidemiological study designs, including ecological, \\ case-control or cohort study. \\ 6. English language publications.
}

${ }^{\dagger}$ The wildcard $\left(^{*}\right)$ was used to identify any other characters.

(2 studies) as there were insufficient studies reporting on kidney cancer incidence.

Combined risk estimates from studies reporting on standardized mortality ratios (SMR) were modeled using a least squares linear regression model for the logged SMRs; studies reporting mortality rates or relative risk (RR - incidence data only) were analyzed with a Generalized Linear Model having a Gamma-distributed response and a log link function, a combination well suited to analyses with highly variable risk estimates [97]. Risk estimates were modeled as a function of logged As and a categorical variable with a level for each study. The latter accounted for possible variations in baseline risk between studies due to differing methodological designs, study quality, populations, etc., and was assumed to be a fixed effect (herein, referred to as Model I, see Boreinsteign et al. [98]). The robustness/sensitivity of the predicted risk estimates obtained with the fixed effects As-risk models was assessed with bootstrap randomizations $(10,000$ permutations) which estimated the effect size at 10,50 and $150 \mu \mathrm{g} / \mathrm{L}$ of As in drinking water (herein, referred to as Model II, see Efron and Tibshirani [99]). A random effects assumption was also examined; however, the small number of studies entering each model precluded a stable estimation of the variance components. Meta-analyses (Model I and II) modeling SMR and RR were only performed for bladder cancer due to the limited number of studies reporting on kidney cancer. Inference of risk at 10, 50 and $150 \mu \mathrm{g} / \mathrm{L}$ of As in drinking water and based on Model I, was only possible for bladder cancer incidence for which a reliable referent population and sufficient number of studies were available. Finally, the effect of sex and smoking on cancer risk was examined; however, analyses could not be completed due to insufficient degrees of freedom. Six of the 7 studies included in the meta-analysis of the RR had been adjusted for tobacco smoking in the original publication - an important risk factor in the development of urinary tract cancers and a possible effect modifier in the cancer-As relationship [51,86,100]. Only one of the 8 studies included in the analyses of the SMR adjusted for smoking [34], as these were generally ecological studies with no individual-level information on smoking. A list of covariates assesses in the original publication appear on Tables 3, 4, 6. Analyses were performed using R 2.13.0 [101].

\section{Results}

\section{Study characteristics}

The search resulted in the review of 249 abstracts, with 50 studies being retained for full text review (Figure 1). In total, forty studies met the inclusion criteria (principally, As in drinking water, toenail or urine as exposure measure and urinary tract cancer as outcome of interest) as listed in Table 1 . Of these, 20 were ecological, 11 were case-control and 9 were cohort epidemiological studies. Thirty-seven of the 40 studies reported on bladder cancer outcomes and of these, 13 also reported on kidney cancer outcomes. One study focused exclusively on kidney cancer mortality [61]. Seventeen studies qualified for inclusion in the meta-analysis, 7 reporting on bladder cancer incidence and 10 on bladder cancer mortality. Two studies also reported on kidney cancer mortality, which was analysed independently from bladder cancer outcomes. Metrics of exposure included: As in well drinking water (median, average or range), cumulative As exposure, years of artesian well water consumption and As in toenails or urine. When measured in drinking water, exposure covered a broad spectrum of As concentrations, ranging from the study-specific detection limit to over $3,500 \mu \mathrm{g} / \mathrm{L}$ and with most study areas showing levels exceeding the WHO advisory limit (Figure 2). Adverse cancer outcomes were reported over the entire range of concentrations, although more consistently in regions where exposure levels were high, typically above $150 \mathrm{ug} / \mathrm{L}$ (Figure 2).

\section{Quality assessment}

The quality of the studies was variable. For examples, all ecological studies assessed As exposure using group level (median or average) or ecologic measurements of drinking 
Table 2 Summary results from ecological studies reporting on arsenic exposure and the risk of bladder cancer

\begin{tabular}{|c|c|c|c|c|c|c|c|}
\hline $\begin{array}{l}\text { Study [reference] } \\
\text { (Table from original } \\
\text { publication) }\end{array}$ & Study locale & Outcome & Exposure $^{1}$ [comments] & $I^{2} D^{2}$ & $\begin{array}{l}\text { Outcome } \\
\text { measure }\end{array}$ & Cases & $\begin{array}{l}\text { Risk estimate } \\
(95 \% \mathrm{Cl})\end{array}$ \\
\hline \multirow[t]{3}{*}{ Chen et al. $1985^{3}$ [24] } & \multirow{3}{*}{$\begin{array}{l}84 \text { villages from } 4 \\
\text { neighbouring townships } \\
\text { on SW coast, Taiwan }\end{array}$} & \multirow[t]{3}{*}{ Mortality 1968-82 } & \multirow{2}{*}{$\begin{array}{l}\text { Median arsenic content of artesian well and (range): } \\
780 \mu \mathrm{\mu g} \cdot \mathrm{L}^{-1}(350-1,140) \text {; in shallow well: } 40(0.0-300) \text {. } \\
\text { Period of samples collection not reported. }\end{array}$} & \multirow[t]{3}{*}{ ICD 188} & \multirow{3}{*}{$\begin{array}{l}\mathrm{SMR} \text { male } \\
\mathrm{SMR}_{\text {female }}\end{array}$} & \multirow{3}{*}{$\begin{array}{l}167 \\
165\end{array}$} & \multirow{3}{*}{$\begin{array}{l}11.0(9.33-12.7) \\
20.1(17.0-23.2)\end{array}$} \\
\hline & & & & & & & \\
\hline & & & $\begin{array}{l}\text { [Comparison of mortality rate in Blackfoot } \\
\text { disease-endemic areas (BFD) with those of the } \\
\text { general population.] }\end{array}$ & & & & \\
\hline \multirow[t]{11}{*}{$\begin{array}{l}\text { *Chen et al. } 1988^{4} \\
\text { [26] (Table One) }\end{array}$} & \multirow[t]{11}{*}{$\begin{array}{l}\text { BFD endemic area, } \\
\text { Taiwan }\end{array}$} & \multirow[t]{11}{*}{ Mortality 1973-86 } & $\begin{array}{l}\text { Arsenic well water concentration }\left(\mu \mathrm{g} \cdot \mathrm{L}^{-1}\right) \text {. Period of } \\
\text { samples collection not reported. }\end{array}$ & \multirow[t]{11}{*}{ ICD9 188} & & & \\
\hline & & & General population & & $\mathrm{ASMR}_{\text {male }}$ & & \\
\hline & & & $<300$ & & & - & 3.1 \\
\hline & & & $300-590$ & & & - & 15.7 \\
\hline & & & \multirow[t]{2}{*}{$\geq 600$} & & & - & 37.8 \\
\hline & & & & & & - & 89.1 \\
\hline & & & General population & & $\mathrm{ASMR}_{\text {female }}$ & & \\
\hline & & & $<300$ & & & - & 1.4 \\
\hline & & & $300-590$ & & & - & 16.7 \\
\hline & & & $\geq 600$ & & & - & 35.1 \\
\hline & & & $\begin{array}{l}\text { [Comparison of mortality rate in BFD with those of } \\
\text { the general population.] }\end{array}$ & & & - & 91.5 \\
\hline \multirow[t]{7}{*}{$\begin{array}{l}\text { *Wu et al. } 1989^{5} \text { [27] } \\
\text { (Table Three) }\end{array}$} & \multirow[t]{7}{*}{$\begin{array}{l}\text { BFD endemic area, } \\
\text { Taiwan (42 villages) }\end{array}$} & \multirow[t]{7}{*}{ Mortality 1973-86 } & $\begin{array}{l}\text { Arsenic well water concentration }\left(\mu \mathrm{g} \cdot \mathrm{L}^{-1}\right) \text { based on } \\
\text { well water samples collected between } 1964-66 \text {. }\end{array}$ & \multirow[t]{7}{*}{ ICD8 188} & & & \\
\hline & & & $<300$ & & $\mathrm{ASMR}_{\text {male }}$ & 23 & 22.6 \\
\hline & & & $300-590$ & & & 36 & 61.0 \\
\hline & & & $\geq 600$ & & & 26 & 92.7 \\
\hline & & & $<300$ & & $\mathrm{ASMR}_{\text {female }}$ & 30 & 25.6 \\
\hline & & & $300-590$ & & & 36 & 57.0 \\
\hline & & & $\geq 600$ & & & 30 & 111.3 \\
\hline \multirow[t]{5}{*}{$\begin{array}{l}\text { Chen and Wang } \\
1990^{6} \text { [28] (Table } \\
\text { Four) }\end{array}$} & \multirow[t]{5}{*}{$\begin{array}{l}314 \text { precincts \& } \\
\text { townships in Taiwan, } \\
\text { including } 4 \text { from BFD } \\
\text { endemic area }\end{array}$} & \multirow[t]{5}{*}{ Mortality 1972-83 } & $\begin{array}{l}\text { Average arsenic levels in water samples of all } 314 \\
\text { geographical units. } 73.9 \% \text { had }<5 \% \text { of wells with } \\
>50 \mu \mathrm{\mu g} \cdot \mathrm{L}^{-1} ; 14.7 \% \text { had } 5-14 \% ; 11.5 \% \text { had } \geq 15 \% \text {. } \\
\text { Well water samples collected between } 1974-76 \text {. }\end{array}$ & \multirow[t]{5}{*}{ ICD 188} & & & \\
\hline & & & \multirow[t]{2}{*}{ All precincts \& townships } & & $\mathrm{ASMR}_{\text {male }}$ & - & $3.9(0.5)$ \\
\hline & & & & & $\mathrm{ASMR}_{\text {female }}$ & - & $4.2(0.5)$ \\
\hline & & & \multirow[t]{2}{*}{ Southwestern townships } & & ASMR male & - & $3.7(0.7)$ \\
\hline & & & & & $\mathrm{ASMR}_{\text {female }}$ & - & $4.5(0.7)$ \\
\hline
\end{tabular}


Table 2 Summary results from ecological studies reporting on arsenic exposure and the risk of bladder cancer (Continued)

\begin{tabular}{|c|c|c|c|c|c|c|c|}
\hline \multirow{12}{*}{$\begin{array}{l}\text { Chiang et al. 19937 } \\
\text { [29] (Table Two) }\end{array}$} & \multirow{12}{*}{$\begin{array}{l}\text { BFD endemic area in } \\
\text { Taiwan and } 2 \\
\text { neighbouring areas }\end{array}$} & \multirow[t]{12}{*}{ Incidence 1981-85 } & \multirow{12}{*}{$\begin{array}{l}\text { Exposure not evaluated, but based on Chen et al. } \\
\text { 1985, the median arsenic content of artesian well in } \\
\text { this area was } 780 \mu \mathrm{g} \cdot \mathrm{L}^{-1}(350-1,140) \text {; that of } \\
\text { shallow well was } 40 \mu \mathrm{g} \cdot \mathrm{L}^{-1}(0.0-300) \text {. Period of } \\
\text { samples collection not reported. } \\
\text { [Comparison of incidence rate in BFD with those of } \\
\text { neighbouring areas and Taiwan as a whole.] }\end{array}$} & \multirow[t]{12}{*}{ N/A } & \multicolumn{3}{|l|}{ Endemic area } \\
\hline & & & & & $\mathbb{R}_{\text {_both_sex }}$ & 140 & 23.5 \\
\hline & & & & & $\mathbb{R}_{\text {male }}$ & 81 & 26.1 \\
\hline & & & & & $I_{\text {female }}$ & 59 & 21.1 \\
\hline & & & & & $\begin{array}{l}\text { Neighbouring } \\
\text { Endemic area }\end{array}$ & & \\
\hline & & & & & IR_both_sex & 13 & 4.45 \\
\hline & & & & & $\mathbb{R}_{\text {male }}$ & 7 & 4.65 \\
\hline & & & & & $\mathbb{R}_{\text {female }}$ & 6 & 4.28 \\
\hline & & & & & All Taiwan & & \\
\hline & & & & & IR_both_sex & 2,135 & 2.29 \\
\hline & & & & & $\mathbb{I}_{\text {male }}$ & 1,608 & 3.31 \\
\hline & & & & & $\mathbb{R}_{\text {female }}$ & 527 & 1.17 \\
\hline $\begin{array}{c}\text { Hopenhayn-Rich et al. } \\
\text { 1996 }{ }^{8} \text { [35] (Table } \\
\text { Three) }\end{array}$ & $\begin{array}{l}26 \text { counties in Cordoba, } \\
\text { Argentina }\end{array}$ & Mortality 1986-91 & $\begin{array}{l}\text { Arsenic drinking water concentration ranging from } \\
100 \text { to } 2,000 \mu \mathrm{g} \cdot \mathrm{L}^{-1} \text {. }\end{array}$ & ICD9 188 & & & \\
\hline \multirow{7}{*}{$\begin{array}{l}\text { *Hopenhayn-Rich } \\
\text { et al. } 1998 \text { [36] (Tables } \\
\text { Three, Four) }\end{array}$} & & & Low & & & 113 & $0.80(0.66-0.96)$ \\
\hline & & & Medium & & $S M R_{\text {male }}$ & 116 & $1.28(1.05-1.53)$ \\
\hline & & & High ( $178 \mu \mathrm{g} \cdot \mathrm{L}^{-1}$ on average) & & & 131 & $2.14(1.78-2.53)$ \\
\hline & & & Low & & & 39 & $1.21(0.85-1.64)$ \\
\hline & & & Medium & & SMR female & 29 & $1.39(0.93-1.99)$ \\
\hline & & & High ( $178 \mu \mathrm{g} \cdot \mathrm{L}^{-1}$ on average) & & & 27 & $1.82(1.19-2.64)$ \\
\hline & & & $\begin{array}{l}\text { [Arsenic measurements from a variety of sources, } \\
\text { including official reports of water analyses from the } \\
\text { 1930, } 2 \text { scientific sampling studies and a water survey.] }\end{array}$ & & & & \\
\hline \multirow[t]{3}{*}{$\begin{array}{l}\text { Guo et al. } 1997^{9} \text { [37] } \\
\text { (Table Two) }\end{array}$} & 243 townships in Taiwan & Incidence 1980-87 & $\begin{array}{l}\text { Arsenic well water concentration ranging from }<50 \\
\text { to }>640 \mu \mathrm{g} \cdot \mathrm{L}^{-1} \text {. }\end{array}$ & ICD 188 & $\mathrm{RD}_{\text {male }}$ & - & $0.57(0.07)$ \\
\hline & & & Estimate presented measured at $>640 \mu \mathrm{g} \cdot \mathrm{L}^{-1}$. & & $\mathrm{RD}_{\text {female }}$ & - & $0.33(0.04)$ \\
\hline & & & $\begin{array}{l}\text { [Arsenic measurements from a National survey of } \\
83,656 \text { wells in } 243 \text { townships, collected mostly } \\
\text { between 1974-76.] }\end{array}$ & & & & \\
\hline \multirow[t]{2}{*}{$\begin{array}{l}\text { Rivara et al.1997 [38] } \\
\quad \text { (Table Four) }\end{array}$} & Chile & Mortality 1950-92 & $\begin{array}{l}\text { Annual average arsenic concentration in drinking } \\
\text { water for Antofagasta (Region II of Chile) ranging } \\
\text { between } 40 \text { to } 860 \mu \mathrm{gg} \cdot \mathrm{L}^{-1} \text {. Data from historical } \\
\text { records from } 1950-1992 \text {. }\end{array}$ & ICD 188 & $\mathrm{RR}$ & - & $10.2(8.6-12.2)$ \\
\hline & & & $\begin{array}{l}\text { [Comparison of mortality rate in Region II (exposed } \\
\text { populations) vs Region VIII (control populations.] }\end{array}$ & & & & \\
\hline
\end{tabular}


Table 2 Summary results from ecological studies reporting on arsenic exposure and the risk of bladder cancer (Continued)

\begin{tabular}{|c|c|c|c|c|c|c|c|}
\hline \multirow[t]{3}{*}{ Smith et al. 1998 [39] } & \multirow[t]{3}{*}{ Chile } & \multirow[t]{3}{*}{ Mortality 1989-93 } & \multirow{3}{*}{$\begin{array}{l}\text { Region II of Northern Chile with population } \\
\text { weighted average arsenic concentration in drinking } \\
\text { water up to } 569 \mu \mathrm{g} \cdot \mathrm{L}^{-1} \text { compared with the rest of } \\
\text { Chile; exposure generally }<10 \mu \mathrm{g} \cdot \mathrm{L}^{-1} \text {. } \\
\text { [Arsenic measurements from } 1950-94 \text {.] }\end{array}$} & \multirow[t]{3}{*}{ N/A } & \multirow{3}{*}{$\begin{array}{l}S M R_{\text {male }} \\
S M R_{\text {female }}\end{array}$} & \multirow{3}{*}{$\begin{array}{l}93 \\
64\end{array}$} & \multirow{3}{*}{$\begin{array}{l}6.0(4.8-7.4) \\
8.2(6.3-10.5)\end{array}$} \\
\hline & & & & & & & \\
\hline & & & & & & & \\
\hline \multirow[t]{2}{*}{$\begin{array}{l}\text { Hinwood et al. } 1999 \\
\text { [88] (Table Two) }\end{array}$} & \multirow[t]{2}{*}{$\begin{array}{l}22 \text { areas in Victoria, } \\
\text { Australia }\end{array}$} & \multirow[t]{2}{*}{ Incidence 1982-91 } & $\begin{array}{l}\text { Median water arsenic concentration ranging } 13 \\
\mu \mathrm{g} \cdot \mathrm{L}^{-1} \text { to } 1,077 \mu \mathrm{g} \cdot \mathrm{L}^{-1} \text {. }\end{array}$ & \multirow[t]{2}{*}{$\begin{array}{l}\text { ICD 188, } \\
189.1-189.3\end{array}$} & \multirow[t]{2}{*}{ SIR } & \multirow[t]{2}{*}{303} & \multirow[t]{2}{*}{$0.94(0.84-1.06)$} \\
\hline & & & $\begin{array}{l}\text { [Selected areas were those where samples with soil } \\
\text { and/or water arsenic concentration were generally } \\
\text { in excess of } 10 \mu \mathrm{\mu g} \cdot \mathrm{L}^{-1} \text {. Period for samples collection } \\
\text { is not available.] }\end{array}$ & & & & \\
\hline \multirow{4}{*}{$\begin{array}{l}\text { *Tsai et al. } 1999[41] \\
\text { (Tables Two, Three) }\end{array}$} & \multirow{4}{*}{$\begin{array}{l}4 \text { townships from BFD } \\
\text { endemic area in SW } \\
\text { coast, Taiwan }\end{array}$} & \multirow[t]{4}{*}{ Mortality 1971-94 } & \multirow{2}{*}{$\begin{array}{l}\text { Median arsenic content of artesian well: } 780 \mu \mathrm{g} \cdot \mathrm{L}^{-1} \\
\text { (range: } 350-1,140 \text { ). Period of samples collection not } \\
\text { reported. Authors state that artesian wells were no } \\
\text { longer used by the mid-1970s. }\end{array}$} & \multirow[t]{4}{*}{ ICD9 188} & SMR Iocal-male & 312 & $8.92(7.96-9.96)$ \\
\hline & & & & & $S M R_{\text {national-male }}$ & 312 & $10.5(9.37-11.7)$ \\
\hline & & & \multirow{2}{*}{$\begin{array}{l}\text { [Comparison of mortality in BFD endemic area with } \\
\text { that of a local reference population (Chiayi-Tainan } \\
\text { county) and that of Taiwan as a whole.] }\end{array}$} & & SMR local-female & 295 & $14.1(12.51-15.8)$ \\
\hline & & & & & $S M R_{\text {national-female }}$ & 295 & $17.8(5.70-19.8)$ \\
\hline \multirow[t]{9}{*}{$\begin{array}{l}\text { *Lamm et al. 2004 } \\
\quad \text { [89] (Table One) }\end{array}$} & \multirow[t]{9}{*}{$\begin{array}{l}133 \text { counties in } 26 \text { states, } \\
\text { USA }\end{array}$} & \multirow[t]{9}{*}{ Mortality 1950-79 } & $\begin{array}{l}\text { Arsenic groundwater water concentration }\left(\mu \mathrm{g} \cdot \mathrm{L}^{-1}\right) \text {. } \\
\text { Period of samples collection not reported. }\end{array}$ & \multirow[t]{9}{*}{ N/A } & \multicolumn{3}{|c|}{ Counties } \\
\hline & & & $3.0-3.9$ & & SMR white_male & 53 & $0.95(0.89-1.01)$ \\
\hline & & & $4.0-4.9$ & & SMR white_male & 22 & $0.95(0.88-1.02)$ \\
\hline & & & $5.0-7.4$ & & SMR white_male & 28 & $0.97(0.85-1.12)$ \\
\hline & & & $7.5-9.9$ & & SMR white_male & 14 & $0.89(0.75-1.06)$ \\
\hline & & & 10.0-19.9 & & SMR white_male & 11 & $0.90(0.78-1.04)$ \\
\hline & & & $20.0-49.9$ & & SMR white_male & 3 & $0.80(0.54-1.17)$ \\
\hline & & & $50.0-59.9$ & & SMR white_male & 2 & $0.73(0.41-1.27)$ \\
\hline & & & $\begin{array}{l}\text { [Median arsenic concentration ranged between 3- } \\
60\left(\mu \mathrm{g} \cdot \mathrm{L}^{-1}\right) \text {, with } 65 \% \text { of the counties and } 82 \% \text { of } \\
\text { the population in the range of } 3-5\left(\mu \mathrm{g} \cdot \mathrm{L}^{-1}\right) \text {.] }\end{array}$ & & & & \\
\hline \multirow{8}{*}{$\begin{array}{l}\text { Marshall et al. } 2007 \\
\text { [50] (Table Three) }\end{array}$} & \multirow[t]{8}{*}{ Chile } & \multirow[t]{8}{*}{ Mortality 1950-2000 } & \multirow{8}{*}{$\begin{array}{l}\text { Northern Chile (Region II) with population weighted } \\
\text { average arsenic concentration in drinking water up } \\
\text { to } 569 \mu \mathrm{\mu g} \cdot \mathrm{L}^{-1} \text { vs Region } V \text { which is otherwise } \\
\text { similar to Region II but not exposed to arsenic. } \\
\text { Between } 1958-1970 \text {, arsenic concentration in water } \\
\text { supply of Antofagasta and nearby Mejillones } \\
\text { (Region II) averaged } 870 \mu \mathrm{g} \cdot \mathrm{L}^{-1} \text { and declined in } \\
\text { the } 1970 \text { s when water treatment plants were } \\
\text { installed. }\end{array}$} & \multirow[t]{8}{*}{ ICD 188} & & & \\
\hline & & & & & $\mathrm{RR}_{\text {male-1971-73 }}$ & 9 & $1.71(0.80-3.69)$ \\
\hline & & & & & RR male-1974-75 & 9 & $5.95(2.22-16.0)$ \\
\hline & & & & & $\mathrm{RR}_{\text {male-1977-79 }}$ & 17 & $2.10(1.19-3.72)$ \\
\hline & & & & & $\mathrm{RR}_{\text {male-1980-82 }}$ & 35 & $5.04(3.13-8.10)$ \\
\hline & & & & & $\mathrm{RR}_{\text {male- }-1983-85}$ & 41 & $5.77(3.66-9.09)$ \\
\hline & & & & & $\mathrm{RR}_{\text {male-1986-88 }}$ & 47 & $6.10(3.97-9.39)$ \\
\hline & & & & & $\mathrm{RR}_{\text {male-1989-91 }}$ & 52 & $4.73(3.23-6.94)$ \\
\hline
\end{tabular}




\begin{tabular}{|c|c|c|c|c|c|c|c|}
\hline & & & & & $R_{\text {male-1992-94 }}$ & 62 & $4.95(3.47-7.06)$ \\
\hline & & & & & $R_{\text {male-1995-97 }}$ & 56 & $4.43(3.07-6.38)$ \\
\hline & & & & & $\mathrm{RR}_{\text {male-1998-2000 }}$ & 58 & $4.27(2.98-6.11)$ \\
\hline & & & & & $\mathrm{RR}_{\text {female-1971-73 }}$ & 7 & $3.45(1.34-8.91)$ \\
\hline & & & & & $\mathrm{RR}_{\text {female-1974-75 }}$ & 4 & $3.09(0.90-10.6)$ \\
\hline & & & & & $\mathrm{RR}_{\text {female-1977-79 }}$ & 10 & $5.39(2.24-13.0)$ \\
\hline & & & & & $\mathrm{RR}_{\text {female- } 1980-82}$ & 22 & $9.10(4.59-18.1)$ \\
\hline & & & & & $\mathrm{RR}_{\text {female-1983-85 }}$ & 22 & $8.41(4.30-16.4)$ \\
\hline & & & & & $\mathrm{RR}_{\text {female-1986- } 88}$ & 37 & $7.28(4.44-12.0)$ \\
\hline & & & & & $\mathrm{RR}_{\text {female-1989-91 }}$ & 35 & $6.61(4.02-10.9)$ \\
\hline & & & & & $\mathrm{RR}_{\text {female-1992-94 }}$ & 42 & $13.8(7.74-24.5)$ \\
\hline & & & & & $\mathrm{RR}_{\text {female-1995-97 }}$ & 44 & $7.60(4.78-12.1)$ \\
\hline & & & & & $\mathrm{RR}_{\text {female-1998-2000 }}$ & 50 & $9.16(5.76-14.5)$ \\
\hline \multirow{2}{*}{$\begin{array}{l}\text { *+Meliker et al. } 2007 \\
\text { [90] (Table Two) }\end{array}$} & \multirow{2}{*}{$\begin{array}{l}6 \text { counties, Southeastern } \\
\text { Michigan, USA }\end{array}$} & \multirow[t]{2}{*}{ Mortality 1979-97 } & \multirow{2}{*}{$\begin{array}{l}\text { Population weighted median arsenic concentration } \\
\text { in water of } 7.58 \mu \mathrm{gg} \cdot \mathrm{L}^{-1} \text {. Data from } 9,251 \text { well water } \\
\text { samples collected between } 1983-2002 \text {. }\end{array}$} & ICD9 188 & $\mathrm{SMR}_{\text {male }}$ & 348 & $0.94(0.82-1.08)$ \\
\hline & & & & & $S M R_{\text {female }}$ & 171 & $0.98(0.80-1.19)$ \\
\hline \multirow[t]{7}{*}{$\begin{array}{l}\text { *+Pou et al. 201112 } \\
\text { [63] (Table Two) }\end{array}$} & \multirow[t]{7}{*}{$\begin{array}{l}26 \text { counties in province } \\
\text { of Cordoba, Argentina }\end{array}$} & \multirow[t]{7}{*}{ Mortality 1986-2006 } & $\begin{array}{l}\text { Arsenic drinking water concentration }\left(\mu \mathrm{g} \cdot \mathrm{L}^{-1}\right) \text {. } \\
\text { Period of samples collection not reported. }\end{array}$ & ICD10 C67 & & & \\
\hline & & & Low (0-40) & & $\mathrm{SMR}_{\text {male }}$ & - & $3.14(2.9-3.4)$ \\
\hline & & & Medium (40-320) & & & - & $4.0(3.6-4.5)$ \\
\hline & & & High $(320-1,800)$ & & & - & $4.7(4.1-5.4)$ \\
\hline & & & Low (0-40) & & $S M R_{\text {female }}$ & - & 1.0 (reference) \\
\hline & & & Medium (40-320) & & & - & $0.94(0.84-1.1)$ \\
\hline & & & $\begin{array}{l}\text { High }(320-1,800) \text { [Arsenic measurements from } \\
\text { many surveys, one dating } 50 \text { years prior to study } \\
\text { publication but with arsenic levels showing high } \\
\text { degree of consistency with a more recent survey } \\
\text { with no exact date detailed.] }\end{array}$ & & & - & $1.2(1.04-1.4)$ \\
\hline $\begin{array}{l}\text { *+Su et al. } 2011 \text { [64] } \\
\text { (Table Two) }\end{array}$ & $\begin{array}{l}\text { BFD endemic area, } \\
\text { Taiwan }\end{array}$ & Mortality 1979-2003 & $\begin{array}{l}\text { Median arsenic content of artesian well: } 780 \mu \mathrm{g} \cdot \mathrm{L}-1 \\
\text { (range: } 350-1,140 \text { ). [Period of samples collection not } \\
\text { reported. Artesian wells in the region were dug in } \\
\text { the } 1920 \text { s but no longer used by mid-1970s. Results } \\
\text { show a comparison of mortality in BFD endemic } \\
\text { area with that of Taiwan.] }\end{array}$ & ICD9 188 & SMR & 785 & $5.3(4.9-5.6)$ \\
\hline \multirow{2}{*}{$\begin{array}{l}\text { †Aballay et al. 2012 } \\
\text { [62] (Table Two) }\end{array}$} & \multirow{2}{*}{$\begin{array}{l}123 \text { districts in province } \\
\text { of Cordoba, Argentina }\end{array}$} & \multirow[t]{2}{*}{ Incidence 2004} & \multirow{2}{*}{$\begin{array}{l}\text { Arsenic water samples from } 3 \text { aquifers: (1) Rjojan } \\
\text { plain (concentration ranged } 0-40 \mu \mathrm{g} \cdot \mathrm{L}^{-1}-23 \text { wells), } \\
\text { (2) Pampean mountains }\left(0-320 \mu \mathrm{g} \cdot \mathrm{L}^{-1}-114 \text { wells) }\right. \\
\text { and (3) Chaco-Pampean plain }\left(0-1,800 \mu \mathrm{g} \cdot \mathrm{L}^{-1}-301\right. \\
\text { wells). In } 80 \text { wells, arsenic was undetected. }\end{array}$} & \multirow[t]{2}{*}{$\mathrm{N} / \mathrm{A}$} & $\mathrm{RR}_{\text {male }}$ & - & $13.8(6.80-28.0)$ \\
\hline & & & & & $R R_{\text {female }}$ & - & $12.7(2.51-63.9)$ \\
\hline
\end{tabular}


Table 2 Summary results from ecological studies reporting on arsenic exposure and the risk of bladder cancer (Continued)

\begin{tabular}{|c|c|c|c|c|c|c|c|}
\hline \multirow{3}{*}{$\begin{array}{l}\text { †Fernández et al. } \\
\quad 2012 \text { [55] }\end{array}$} & \multirow[t]{3}{*}{ Antofagasta, Chile } & \multirow[t]{3}{*}{ Mortality 1983-2009 } & \multirow{3}{*}{$\begin{array}{l}\text { Arsenic drinking water concentration ranging } 800- \\
900 \mathrm{\mu g} \cdot \mathrm{L}^{-1} \text {. [Arsenic levels based on the last } 60 \\
\text { years and obtained from the local tap water } \\
\text { company in Antofagasta. Results compares } \\
\text { mortality rate in Antofagasta with the rest of Chile.] }\end{array}$} & \multirow[t]{3}{*}{ ICD10 C67 } & $\mathrm{RR}_{\text {male }}$ & - & $5.3(4.8-5.8)$ \\
\hline & & & & & $R R_{\text {female }}$ & - & $7.8(7.0-8.7)$ \\
\hline & & & & & $\mathrm{RR}_{\text {both_sex }}$ & - & $6.1(5.7-6.6)$ \\
\hline
\end{tabular}

*Study included in meta-analyses.

tRecent study not included in the International Agency for Research on Cancer 2012 review (Monograph 100C [23]).

TRecent study not included in the International Agency for Research
1 All ecological studies assessed arsenic exposure at the group-level.

${ }^{2} \mathrm{CD}=$ International Classification for Disease for cancer site abstracted which included, bladder and urothelial/transitional cell carcinoma of the bladder or kidney. Transitional cell carcinoma of the renal pelvis often share the same etiology as bladder cancer, and as such, have been treated as bladder within the meta-analyses as recommended by IARC [23]. N/A = not available.

${ }^{3} \mathrm{SMR}$, standardized mortality ratio.

${ }_{5}^{4}$ Age-standardized mortality rates per 100,000 using the 1976 world population as standard population and based on 899,811 person-years.

${ }_{6}^{5}$ All age-standardized mortality rates shown are significant at $\mathrm{p}<0.001$ based on trend test.

${ }^{6}$ Regression coefficient showing an increase in age-adjusted mortality per 100,000 persons-years for every 0.1 ppm increase in arsenic level, adjusting for indices of industrialization and urbanization. Standard errors

are in brackets. Bladder cancer was significantly correlated with average arsenic level in water.

${ }^{7}$ Incidence rate per 100,000, adjusted for age.

${ }^{8}$ County is the unit of analysis.

${ }^{9} \mathrm{RD}$, rate difference (per 100,000 person-years) for one unit increase in the predictor and associated standard error for exposure $>640 \mu \mathrm{gg} \cdot \mathrm{L}^{-1}(\mathrm{SE})$. Results shown for transitional-cell carcinoma.

${ }^{10}$ Average annual age-adjusted (to U.S. 1970 standard population) death rates per 100,000 abstracted at the state level for each decade were used as standard rates to calculate county-specific SMRs.

${ }^{11}$ Incidence rate ratio estimates with arsenic as continuous.

${ }^{12}$ Used lung cancer mortality rates as surrogate to smoking - may result in an overestimation of risk where smoking has declined; an underestimation of risk where smoking has increased; and an over-adjusted model

as lung cancer is also associated with arsenic exposure. 
Table 3 Summary results from case-control studies reporting on arsenic exposure and the risk of bladder cancer

\begin{tabular}{|c|c|c|c|c|c|c|c|c|c|c|c|c|c|}
\hline \multirow{2}{*}{$\begin{array}{l}\text { Study } \\
\text { [reference] } \\
\text { (Table from } \\
\text { original } \\
\text { publication) }\end{array}$} & \multirow[t]{2}{*}{ Study locale } & \multirow[t]{2}{*}{ Outcome } & \multirow[t]{2}{*}{$I^{\prime C D}{ }^{1}$} & \multirow{2}{*}{$\begin{array}{c}\text { Arsenic } \\
\text { exposure } \\
\text { assessment }\end{array}$} & \multirow[t]{2}{*}{ Exposure [comments] } & \multirow[t]{2}{*}{$\begin{array}{l}\text { Cases: } \\
\text { Controls }\end{array}$} & \multicolumn{2}{|c|}{$\begin{array}{c}\text { All } \\
\text { participants }\end{array}$} & \multicolumn{2}{|c|}{$\begin{array}{c}\text { Never } \\
\text { smokers }\end{array}$} & \multicolumn{2}{|c|}{$\begin{array}{c}\text { Ever } \\
\text { smokers }\end{array}$} & \multirow[t]{2}{*}{ Covariates assessed } \\
\hline & & & & & & & $n$ & $\begin{array}{c}\mathrm{OR}^{2} \\
(95 \% \mathrm{Cl})\end{array}$ & $\bar{n}$ & $\begin{array}{c}\text { OR, } \\
(95 \% \mathrm{Cl})\end{array}$ & $\mathrm{n}$ & $\begin{array}{c}\text { OR, } \\
(95 \% \mathrm{Cl})\end{array}$ & \\
\hline \multirow{6}{*}{$\begin{array}{c}\text { Chen et al. } \\
\text { 1986 }{ }^{3} \text { [25] (Table } \\
\text { Four) }\end{array}$} & \multirow{6}{*}{$\begin{array}{l}4 \text { neighbouring } \\
\text { Blackfoot } \\
\text { disease (BFD)- } \\
\text { endemic areas, } \\
\text { Taiwan }\end{array}$} & \multirow[t]{6}{*}{$\begin{array}{l}\text { Mortality } \\
1996-2000\end{array}$} & \multirow[t]{6}{*}{ N/A } & \multirow[t]{6}{*}{$\begin{array}{l}\text { Individual level } \\
\text { 'estimated' }\end{array}$} & $\begin{array}{l}\text { Year of artesian water } \\
\text { consumption: }\end{array}$ & \multirow[t]{6}{*}{$69: 368$} & & & & & & & \multirow{6}{*}{$\begin{array}{l}\text { age, sex, cigarette } \\
\text { smoking, tea drinking } \\
\text { habit, vegetarian habit, } \\
\text { vegetable consumption } \\
\text { frequency, fermented } \\
\text { bean consumption } \\
\text { frequency }\end{array}$} \\
\hline & & & & & 0 (referent) & & 17 & 1.0 & - & - & - & - & \\
\hline & & & & & $1-20$ & & 19 & 1.27 & - & - & - & - & \\
\hline & & & & & $20-40$ & & 10 & 1.68 & - & - & - & - & \\
\hline & & & & & $\geq 40$ & & 23 & 4.10 & - & - & - & - & \\
\hline & & & & & $\begin{array}{l}\text { [Median arsenic content of } \\
\text { artesian wells and (range): } 780 \\
\mu g^{\circ} L^{-1}(350-1,140) \text {. History of } \\
\text { artesian well water noted.] }\end{array}$ & & & & & & & & \\
\hline \multirow{6}{*}{$\begin{array}{c}\text { Bates et al. } 1995 \\
\text { [31] (Table } \\
\text { Three) }\end{array}$} & \multirow{6}{*}{ Utah, USA } & Incidence & \multirow[t]{6}{*}{ N/A } & \multirow[t]{6}{*}{$\begin{array}{l}\text { Individual level } \\
\text { 'measured' }\end{array}$} & $\begin{array}{l}\text { Cumulative dose index of } \\
\text { arsenic (mg): }\end{array}$ & \multirow[t]{6}{*}{ 117:266 } & & & & & & & \multirow{6}{*}{$\begin{array}{l}\text { age, sex, smoking, } \\
\text { exposure to chlorinated } \\
\text { surface water, history of } \\
\text { bladder infection, } \\
\text { education, urbanization of } \\
\text { the place of longest } \\
\text { lifetime residence, and } \\
\text { ever employed in high- } \\
\text { risk occupation }\end{array}$} \\
\hline & & \multirow{5}{*}{$\begin{array}{l}\text { Diagnosis in a } \\
1 \text {-year period } \\
\text { around } 1978\end{array}$} & & & $<19$ (referent) & & 14 & 1.0 & 10 & 1.0 & 4 & 1.0 & \\
\hline & & & & & 19 to $<33$ & & 21 & $\begin{array}{c}1.56 \\
(0.8-3.2)\end{array}$ & 10 & $\begin{array}{c}1.09 \\
(0.4-3.1)\end{array}$ & 11 & $\begin{array}{c}3.33 \\
(1.0-10.8)\end{array}$ & \\
\hline & & & & & 33 to $<53$ & & 17 & $\begin{array}{c}0.95 \\
(0.4-2.0)\end{array}$ & 7 & $\begin{array}{c}0.68 \\
(0.2-2.3)\end{array}$ & 10 & $\begin{array}{c}1.93 \\
(0.6-6.2)\end{array}$ & \\
\hline & & & & & $\geq 53$ & & 19 & 1.41 & 4 & 0.53 & 15 & 3.32 & \\
\hline & & & & & $\begin{array}{l}\text { [Arsenic water concentration } \\
\text { ranged } 0.5-160 \mu \mathrm{g} \cdot \mathrm{L} \text { and av- } \\
\text { eraged } 5 \mu \mathrm{g} \cdot \mathrm{L} \text {. Data on arsenic } \\
\text { levels in public drinking water } \\
\text { supplies were collected in } \\
1978-79 \text {. Results are based on } \\
\text { the } 71 \text { cases who had lived in } \\
\text { study towns for at least half of } \\
\text { their lives. Residential history } \\
\text { and water source used in ex- } \\
\text { posure assessment.] }\end{array}$ & & & & & & & & \\
\hline \multirow{5}{*}{$\begin{array}{c}\text { *Kurttio et al. } \\
1999 \text { [20] (Tables } \\
\text { Six, Seven) }\end{array}$} & \multirow{5}{*}{$\begin{array}{l}\text { Areas in Finland } \\
\text { with }<10 \% \\
\text { population with } \\
\text { municipal } \\
\text { drinking-water } \\
\text { system }\end{array}$} & \multirow[t]{5}{*}{$\begin{array}{l}\text { Incidence } \\
\text { 1981-95 }\end{array}$} & \multirow[t]{5}{*}{ N/A } & \multirow[t]{5}{*}{$\begin{array}{l}\text { Individual level } \\
\text { 'measured' }\end{array}$} & $\begin{array}{l}\text { Arsenic water concentration } \\
\left(\mu \mathrm{g}^{\circ} \cdot \mathrm{L}^{-1}\right) \text { : }\end{array}$ & $61: 275$ & & & & & & & \multirow[t]{5}{*}{ age, sex, smoking } \\
\hline & & & & & $<0.1$ & & 23 & 1.0 & 8 & 1.0 & 8 & 1.0 & \\
\hline & & & & & $1.1-0.5$ & & 19 & $\begin{array}{c}1.53 \\
(0.75-3.09)\end{array}$ & 4 & $\begin{array}{c}0.95 \\
(0.25-3.64)\end{array}$ & 3 & $\begin{array}{c}1.10 \\
(0.19-6.24)\end{array}$ & \\
\hline & & & & & $\geq 0.5$ & & 19 & $\begin{array}{c}2.44 \\
(1.11-5.37)\end{array}$ & 5 & $\begin{array}{c}0.87 \\
(0.25-3.02)\end{array}$ & 7 & $\begin{array}{c}10.3 \\
(1.16-92.6)\end{array}$ & \\
\hline & & & & & (log) continuous & & 61 & $\begin{array}{c}1.37 \\
(0.95-1.96)\end{array}$ & & - & & - & \\
\hline
\end{tabular}

Bates et al. 1995 Utah, USA
[31] (Table 31] (Table
Three)

*Kurttio et a population $n$ municipal drinking-water 
Table 3 Summary results from case-control studies reporting on arsenic exposure and the risk of bladder cancer (Continued)

Chen et al. 2003 Southwestern [91] (Table Two) Taiwan

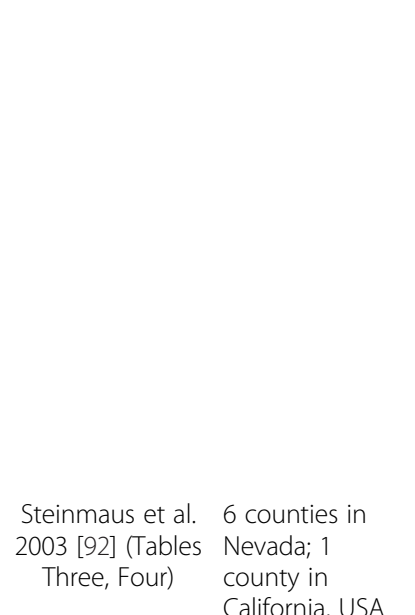

Incidence 1996-99 Incidence
1994-2000 California, USA
[Only subjects with drilled

wells; median total arsenic

concentration of $0.1 \mu \mathrm{g} \cdot \mathrm{L}$;

max.concentration of $64 \mu \mathrm{g} \cdot \mathrm{L}$

and $1 \%$ exceeding $10 \mu \mathrm{g} \cdot \mathrm{L}$.

Water sampled from wells used

by the study population at

least for 1967-80. Exposure in

the 3rd-9th calendar year prior

to cancer diagnosis. Residentia

history and drinking water con-

sumption used in exposure

assessment.]

Cumulative arsenic exposure

49:224

CD9 Individual level Cumulative ars

$0-2$

$>2-12$

$>12$

[Arsenic concentration in

artesian well water from survey of 83,656 wells between 1974 76. Questionnaires used to determine village in which subjects lived 30 years ago. Residential history and duration and: source of drinking wat used in exposure assessment]

N/A Individual level Cumulative exposure to arsenic 181:328 'estimated' in water $\left(\mathrm{mg} \cdot \mathrm{L}^{-1} \cdot\right.$ year):

$$
<6.4
$$

$6.4-82.8$

$>82.8$

[Arsenic concentration from 7,000 samples from community and domestic wells. Results for a 40 years lagged exposure; a 40 years lagged exposure;
$88.4 \%$ of cases and $91.8 \%$ of $88.4 \%$ of cases and $91.8 \%$ o
controls being exposed to arsenic levels ranging from 0 to $19 \mu \mathrm{g} \cdot \mathrm{L}$, respectively.

Residential history, source of

drinking water and intake used in exposure assessment.] age, sex, BMl, cumulative arsenic exposure,

cigarette smoking, hair

30

4

$(-1.1-3.0)$

$10 \quad 1.86$

$(0.2-5.10)$
OR for all participants

adjusted for age, gender,

$\begin{array}{lllllll}153 & 1.0 & 23 & 1.0 & 130 & 1.0 & \begin{array}{l}\text { occupation, smoking } \\ \text { history }(<1 \text { pack per day }\end{array}\end{array}$ $\begin{array}{lllllll}1.63 & 3 & 2.65 & 6 & 1.06 & (\mathrm{ppd}), \geq 1 \mathrm{ppd}, \text { former }\end{array}$

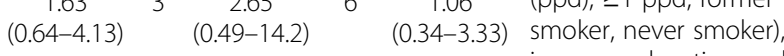

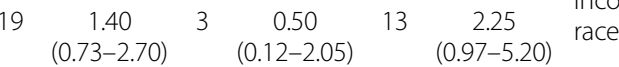


Table 3 Summary results from case-control studies reporting on arsenic exposure and the risk of bladder cancer (Continued)

\begin{tabular}{|c|c|c|c|c|c|c|c|c|c|c|c|c|c|}
\hline \multirow{6}{*}{$\begin{array}{c}\text { *Bates et al. } \\
2004 \text { [93] (Tables } \\
\text { Two, Three) }\end{array}$} & \multirow{6}{*}{$\begin{array}{l}\text { Cordoba, } \\
\text { Argentina }\end{array}$} & \multirow[t]{6}{*}{$\begin{array}{l}\text { Incidence } \\
\text { 1996-2000 }\end{array}$} & \multirow[t]{6}{*}{ N/A } & \multirow[t]{6}{*}{$\begin{array}{l}\text { Individual level } \\
\text { 'measured' }\end{array}$} & $\begin{array}{l}\text { Arsenic water concentration } \\
\left(\mu g^{\circ} \cdot L^{-1}\right):\end{array}$ & \multirow[t]{6}{*}{ 114:114 } & & & & & & & \multirow{6}{*}{$\begin{array}{l}\text { mate con bombilla } \\
\text { consumption, education, } \\
\text { and home tap-water con- } \\
\text { sumption in all groups; } \\
\text { and adjusted for the } \\
\text { highest daily number of } \\
\text { cigarettes subjects re- } \\
\text { ported ever having } \\
\text { smoked in the smoker } \\
\text { group }\end{array}$} \\
\hline & & & & & $0-50$ & & 70 & 1.0 & 22 & 1.0 & 65 & 1.0 & \\
\hline & & & & & $51-100$ & & 13 & $\begin{array}{c}0.88 \\
(0.3-2.3)\end{array}$ & 2 & $\begin{array}{c}1.05 \\
(0.2-6.9)\end{array}$ & 7 & $\begin{array}{c}1.29 \\
(0.3-5.0)\end{array}$ & \\
\hline & & & & & $101-200$ & & 22 & $\begin{array}{c}1.02 \\
(0.5-2.3)\end{array}$ & 3 & $\begin{array}{c}1.10 \\
(0.2-6.3)\end{array}$ & 10 & $\begin{array}{c}0.96 \\
(0.3-3.0)\end{array}$ & \\
\hline & & & & & $>200$ & & 9 & 0.60 & 1 & 0.58 & 2 & 0.17 & \\
\hline & & & & & $\begin{array}{l}\text { [Average arsenic concentration } \\
\text { of } 5 \text { years of highest exposure } \\
\text { during the period } 6-40 \text { years } \\
\text { before interview. On average, } \\
\text { cases and controls had } 25.7 \\
\text { and } 25.6 \text { years of well-water } \\
\text { consumption, respectively; also } \\
\text { approximately } 50 \% \text { of all well } \\
\text { years were derived from proxy- } \\
\text { well data. Results shown for } \\
\text { transitional cell bladder cancer.] }\end{array}$ & & & & & & & (0.0-1.0) & \\
\hline \multirow{9}{*}{$\begin{array}{c}\text { Karagas et al. } \\
2004 \text { [94] (Table } \\
\text { Two) }\end{array}$} & \multirow[t]{9}{*}{$\begin{array}{l}\text { New Hampshire, } \\
\text { USA }\end{array}$} & \multirow[t]{9}{*}{$\begin{array}{l}\text { Incidence } \\
\text { 1994-98 }\end{array}$} & \multirow[t]{9}{*}{$\mathrm{N} / \mathrm{A}$} & \multirow[t]{9}{*}{$\begin{array}{l}\text { Individual level } \\
\text { 'measured' }\end{array}$} & $\begin{array}{l}\text { Arsenic toenail concentration } \\
\left(\mu g^{\prime} \cdot g^{-1}\right) \text { : }\end{array}$ & \multirow[t]{9}{*}{ 383:641 } & & & & & & & \multirow[t]{9}{*}{$\begin{array}{l}\text { age, sex, smoking status } \\
\text { (ever/never) }\end{array}$} \\
\hline & & & & & $0.009-0.059$ & & 90 & 1.0 & 15 & 1.0 & 75 & 1.0 & \\
\hline & & & & & $0.060-0.086$ & & 119 & $\begin{array}{c}1.37 \\
(0.96-1.96)\end{array}$ & 20 & $\begin{array}{c}0.85 \\
(0.38-1.91)\end{array}$ & 99 & $\begin{array}{c}1.53 \\
(1.02-2.29)\end{array}$ & \\
\hline & & & & & $0.087-0.126$ & & 88 & $\begin{array}{c}1.08 \\
(0.74-1.58)\end{array}$ & 22 & $\begin{array}{c}1.18 \\
(0.53-2.66)\end{array}$ & 66 & $\begin{array}{c}1.02 \\
(0.66-1.56)\end{array}$ & \\
\hline & & & & & $0.127-0.193$ & & 48 & $\begin{array}{c}1.04 \\
(0.66-1.63)\end{array}$ & 11 & $\begin{array}{c}1.10 \\
(0.42-2.90)\end{array}$ & 37 & $\begin{array}{c}1.00 \\
(0.60-1.67)\end{array}$ & \\
\hline & & & & & $0.194-0.277$ & & 2 & $\begin{array}{c}1.33 \\
(0.71-2.49)\end{array}$ & 3 & $\begin{array}{c}0.49 \\
(0.12-2.05)\end{array}$ & 18 & $\begin{array}{c}1.78 \\
(0.86-3.67)\end{array}$ & \\
\hline & & & & & $0.278-0.330$ & & 3 & $\begin{array}{c}0.41 \\
(0.11-1.50)\end{array}$ & 0 & - & 3 & $\begin{array}{c}0.50 \\
(0.13-1.88)\end{array}$ & \\
\hline & & & & & $0.331-2.484$ & & \multirow[t]{2}{*}{14} & \multirow{2}{*}{$\begin{array}{c}1.36 \\
(0.63-2.90)\end{array}$} & \multirow[t]{2}{*}{0} & \multirow[t]{2}{*}{-} & \multirow[t]{2}{*}{14} & \multirow{2}{*}{$\begin{array}{c}2.17 \\
(0.92-5.11)\end{array}$} & \\
\hline & & & & & $\begin{array}{l}\text { [Levels of arsenic in toenails } \\
\text { reflect exposures occurring } \\
\text { between } 9-15 \text { months prior to } \\
\text { sample collection. On average } \\
\text { cases and controls had } 16.5 \\
\text { and } 17.2 \text { years exposure to } \\
\text { their water system. Results } \\
\text { shown for transitional cell } \\
\text { bladder cancer.] }\end{array}$ & & & & & & & & \\
\hline
\end{tabular}


Table 3 Summary results from case-control studies reporting on arsenic exposure and the risk of bladder cancer (Continued)

\begin{tabular}{|c|c|c|c|c|c|c|c|c|c|c|c|c|c|}
\hline \multirow{6}{*}{$\begin{array}{c}\text { Michaud et al. } \\
2004 \text { [95] (Table } \\
\text { Two) }\end{array}$} & \multirow{6}{*}{$\begin{array}{l}\text { Southwestern } \\
\text { Finland }\end{array}$} & \multirow[t]{6}{*}{$\begin{array}{l}\text { Incidence } \\
\text { 1985-99 }\end{array}$} & \multirow{6}{*}{$\begin{array}{c}\text { ICD9 } \\
188 \\
233.7\end{array}$} & \multirow{6}{*}{$\begin{array}{l}\text { Individual level } \\
\text { 'measured' }\end{array}$} & $\begin{array}{l}\text { Arsenic toenail concentration } \\
\left(\mu g^{\prime} \cdot g^{-1}\right) \text { : }\end{array}$ & \multirow[t]{6}{*}{$280: 293$} & & & & & & & \multirow{6}{*}{$\begin{array}{l}\text { age, toenail collection } \\
\text { date, intervention group, } \\
\text { number of cigarettes per } \\
\text { day, and number of } \\
\text { years smoking }\end{array}$} \\
\hline & & & & & $<0.105$ & & - & - & - & - & 136 & 1.0 & \\
\hline & & & & & $0.105-0.160$ & & - & - & - & - & 73 & $\begin{array}{c}1.10 \\
(0.73-1.64)\end{array}$ & \\
\hline & & & & & $0.161-0.259$ & & - & - & - & - & 37 & $\begin{array}{c}0.93 \\
(0.56-1.54)\end{array}$ & \\
\hline & & & & & $0.260-0.399$ & & - & - & - & - & 20 & $\begin{array}{c}1.38 \\
(0.68-2.80)\end{array}$ & \\
\hline & & & & & $>0.399$ & & - & - & - & - & 14 & $\begin{array}{c}1.14 \\
(0.52-2.51)\end{array}$ & \\
\hline \multirow{7}{*}{$\begin{array}{l}\text { † Pu et al. } 2007 \\
\text { [51] (Tables } \\
\text { Four, Five) }\end{array}$} & \multirow[t]{7}{*}{ Taiwan } & \multirow[t]{7}{*}{$\begin{array}{l}\text { Incidence } \\
\text { 2002-04 }\end{array}$} & \multirow[t]{7}{*}{$\mathrm{N} / \mathrm{A}$} & \multirow[t]{7}{*}{$\begin{array}{l}\text { Individual level } \\
\text { 'measured' }\end{array}$} & $\begin{array}{l}\text { Arsenic urine concentration } \\
\left(\mu g^{\prime} \cdot g^{-1} \text { creatine): }\right.\end{array}$ & \multirow[t]{7}{*}{ 177:313 } & & & & & & & \multirow{4}{*}{$\begin{array}{l}\text { OR (all participants): age, } \\
\text { sex, education, parents' } \\
\text { ethnicity, alcohol } \\
\text { drinking, pesticides use }\end{array}$} \\
\hline & & & & & $\leq 15.4$ & & 24 & 1.0 & - & - & - & - & \\
\hline & & & & & $15.5-26.4$ & & 44 & $\begin{array}{c}1.9 \\
(1.1-3.4)\end{array}$ & - & - & - & - & \\
\hline & & & & & $>26.4$ & & 109 & $\begin{array}{c}5.3 \\
(3.1-9.0)\end{array}$ & - & - & - & - & \\
\hline & & & & & $\leq 20.3$ & & - & - & 17 & 1.0 & 21 & 1.0 & \multirow{3}{*}{$\begin{array}{l}\text { OR (never/ever smokers): } \\
\text { age, sex }\end{array}$} \\
\hline & & & & & $\geq 20.3$ & & - & - & 68 & 4.4 & 61 & 8.2 & \\
\hline & & & & & $\begin{array}{l}\text { [Smokers include current and } \\
\text { former smokers. Non-smokers } \\
\text { with } \leq 20.3\left(\mu \mathrm{g} \cdot \mathrm{g}^{-1} \text { creatine) }\right. \\
\text { was used as referent category.] }\end{array}$ & & & & & $(2.3-8.5)$ & & $(3.8-1 / .8)$ & \\
\hline \multirow{5}{*}{$\begin{array}{c}\text { *+Meliker et al. } \\
2010 \text { [87] (Table } \\
\text { Three) }\end{array}$} & \multirow{5}{*}{$\begin{array}{l}11 \text { counties of } \\
\text { Southeastern } \\
\text { Michigan, USA }\end{array}$} & \multirow[t]{5}{*}{$\begin{array}{l}\text { Incidence } \\
\text { 2000-04 }\end{array}$} & \multirow[t]{5}{*}{ N/A } & \multirow[t]{5}{*}{$\begin{array}{l}\text { Individual level } \\
\text { 'measured' }\end{array}$} & $\begin{array}{l}\text { Arsenic water concentration } \\
\left(\mu g^{\circ} \cdot L^{-1}\right):\end{array}$ & \multirow[t]{5}{*}{$411: 566$} & & & & & & & \multirow{5}{*}{$\begin{array}{l}\text { age, sex, race, smoking } \\
\text { history, education, history } \\
\text { of employment in high } \\
\text { risk occupation, family } \\
\text { history of bladder cancer }\end{array}$} \\
\hline & & & & & $<1$ & & 187 & 1.0 & - & - & - & - & \\
\hline & & & & & $1-10$ & & 182 & $\begin{array}{c}0.84 \\
(0.63-1.12)\end{array}$ & - & - & - & - & \\
\hline & & & & & $>10$ & & 38 & 1.10 & - & - & - & - & \\
\hline & & & & & $\begin{array}{l}\text { [Arsenic water concentrations } \\
\text { obtained from: 6,050 private } \\
\text { untreated wells sampled } \\
\text { between 1993-2002; } 371 \text { well } \\
\text { water measurements from } \\
\text { participants' current residence } \\
\text { and; } 1,675 \text { measurements from } \\
\text { public well water supplies } \\
\text { collected between 1983-2004, } \\
\text { which were used to estimate } \\
\text { arsenic concentrations at past } \\
\text { residences.] }\end{array}$ & & & $(0.65-1.86)$ & & & & & \\
\hline
\end{tabular}


Table 3 Summary results from case-control studies reporting on arsenic exposure and the risk of bladder cancer (Continued)

\begin{tabular}{|c|c|c|c|c|c|c|c|c|c|c|c|c|c|}
\hline \multirow{6}{*}{$\begin{array}{c}\text { *tSteinmaus } \\
\text { et al. } 2013 \text { [67] } \\
\text { (Table Two) }\end{array}$} & \multirow[t]{6}{*}{$\begin{array}{l}\text { Region I and II, } \\
\text { northern Chile }\end{array}$} & \multirow[t]{6}{*}{$\begin{array}{l}\text { Incidence } \\
\text { 2007-10 }\end{array}$} & \multirow[t]{6}{*}{ N/A } & \multirow[t]{6}{*}{$\begin{array}{l}\text { Individual level } \\
\text { 'estimated' }\end{array}$} & $\begin{array}{l}\text { Arsenic water concentration } \\
\left(\mu g \cdot L^{-1}\right) \text { : }\end{array}$ & $306: 640$ & & & & & & & \multirow{6}{*}{$\begin{array}{l}\text { no covariates assessed, } \\
\text { although subjects were } \\
\text { frequency matched on } \\
\text { age, sex }\end{array}$} \\
\hline & & & & & $0-59$ & & 23 & 1.0 & - & - & - & - & \\
\hline & & & & & 60-199 & & 27 & $\begin{array}{c}0.84 \\
(0.46-1.52)\end{array}$ & - & - & - & - & \\
\hline & & & & & 200-799 & & 60 & $\begin{array}{c}2.50 \\
(1.48-4.22)\end{array}$ & - & - & - & - & \\
\hline & & & & & $>800$ & & \multirow[t]{2}{*}{122} & \multirow{2}{*}{$\begin{array}{c}4.44 \\
(2.75-7.15)\end{array}$} & \multirow[t]{2}{*}{ - } & \multirow[t]{2}{*}{-} & \multirow[t]{2}{*}{-} & \multirow[t]{2}{*}{-} & \\
\hline & & & & & $\begin{array}{l}\text { [Each city/town of residence in } \\
\text { which each subject lived was } \\
\text { linked to a water arsenic } \\
\text { measurement for that city/ } \\
\text { town so that an arsenic } \\
\text { concentration could be } \\
\text { assigned to each year of each } \\
\text { subject's life. Study also present } \\
\text { OR in relation to various } \\
\text { metrics of arsenic exposure } \\
\text { such as lifetime and cumulative } \\
\text { average exposure and; lifetime } \\
\text { and cumulative intake. } \\
\text { Residential history used in } \\
\text { exposure assessment.] }\end{array}$ & & & & & & & & \\
\hline
\end{tabular}

*Study included in meta-analyses.

†Recent study not included in the International Agency for Research on Cancer 2012 review (Monograph 100C [23]). ${ }^{1} \mathrm{ICD}=$ International Classification of Disease. N/A = not available.

${ }^{2} \mathrm{OR}=$ Odds ratios.

${ }^{3} \mathrm{OR}$ crude $=1.0,1.17,1.60,3.90$ for corresponding years of exposure shown in table. 
Table 4 Summary results from cohort studies reporting on arsenic exposure and the risk of bladder cancer

\begin{tabular}{|c|c|c|c|c|c|c|c|c|c|c|}
\hline $\begin{array}{l}\text { Study [reference] } \\
\text { (Table from } \\
\text { original } \\
\text { publication) }\end{array}$ & Study locale & Outcome & ICD1 & $\begin{array}{c}\text { Arsenic } \\
\text { exposure } \\
\text { assessment }\end{array}$ & Exposure [comments] & $\begin{array}{l}\text { Outcome } \\
\text { measure }\end{array}$ & $\begin{array}{l}\text { Cohort } \\
\text { size }\end{array}$ & Cases & $\begin{array}{c}\text { Risk } \\
\text { estimate } \\
(95 \% \mathrm{Cl})\end{array}$ & Covariates assessed \\
\hline $\begin{array}{l}\text { Chen et al. } 1988 \\
\text { [70] (Table Six) }\end{array}$ & $\begin{array}{l}4 \text { neighbouring } \\
\text { townships from } \\
\text { Blackfoot disease } \\
\text { (BFD) endemic area, } \\
\text { Taiwan }\end{array}$ & Morality 1968-83 & N/A & Group level & $\begin{array}{l}\text { Median arsenic content } \\
\text { of artesian well and } \\
\text { (range): } 0.78 \mathrm{ppm}(0.35- \\
\text { 1.14); in shallow well: } 0.04 \\
\text { (0.00-0.30). General } \\
\text { population used as } \\
\text { reference. } 95 \% \text { CI } \\
\text { obtained from IARC } 2012 \\
\text { review [23]. }\end{array}$ & SMR & 871 & 15 & $\begin{array}{c}38.8 \\
(21.7-64.0)\end{array}$ & \\
\hline \multirow[t]{6}{*}{$\begin{array}{l}\text { Chiou et al. } 1995 \\
\text { [32] (Table Four) }\end{array}$} & \multirow{6}{*}{$\begin{array}{l}4 \text { neighbouring } \\
\text { townships from BFD } \\
\text { endemic area, Taiwan }\end{array}$} & \multirow{6}{*}{$\begin{array}{l}\text { Incidence } 1988 \\
\text { (Follow-up period } \\
\text { ranged } 0.05 \text { to } 7.7 \\
\text { years) }\end{array}$} & \multirow[t]{6}{*}{ N/A } & \multirow[t]{6}{*}{$\begin{array}{l}\text { Individual level } \\
\text { 'estimated' }\end{array}$} & $\begin{array}{l}\text { Cumulative arsenic } \\
\text { exposure }\left(\mathrm{mg} \cdot \mathrm{L}^{-1} \cdot \text { year): }\right.\end{array}$ & \multirow[t]{6}{*}{ RR } & \multirow[t]{6}{*}{2,556} & 29 & & \multirow[t]{6}{*}{$\begin{array}{l}\text { age, sex, cigarette } \\
\text { smoking }\end{array}$} \\
\hline & & & & & 0 & & & & 1.0 & \\
\hline & & & & & $0.1-19.9$ & & & & $\begin{array}{c}1.57 \\
(0.44-5.55)\end{array}$ & \\
\hline & & & & & $>20$ & & & & $\begin{array}{c}3.58 \\
(1.05-12.19)\end{array}$ & \\
\hline & & & & & unknown & & & & 1.25 & \\
\hline & & & & & $\begin{array}{l}\text { [Median arsenic content } \\
\text { of artesian well and } \\
\text { (range): } 0.78 \mathrm{ppm}(0.35- \\
1.14) \text {; in shallow well: } 0.04 \\
(0.00-0.30) \text {. Histories of } \\
\text { residential address and } \\
\text { duration of drinking well } \\
\text { water used to derive } \\
\text { cumulative exposure.] }\end{array}$ & & & & $(0.38-4.12)$ & \\
\hline \multirow{5}{*}{$\begin{array}{c}\text { *Tsuda et al. } \\
1995 \text { [34] (Table } \\
\text { Three) }\end{array}$} & \multirow[t]{5}{*}{ Niigata, Japan } & \multirow{5}{*}{$\begin{array}{l}\text { Mortality 1959-92 (Re- } \\
\text { cruitment in 1959, } \\
\text { followed until 1992) }\end{array}$} & \multirow[t]{3}{*}{$\begin{array}{l}\text { Transitional } \\
\text { cell carcinoma }\end{array}$} & \multirow[t]{5}{*}{$\begin{array}{l}\text { Individual level } \\
\text { 'measured' }\end{array}$} & $\begin{array}{l}\text { Arsenic water } \\
\text { concentration }\left(\mu \mathrm{g} \cdot L^{-1}\right) \text { : }\end{array}$ & \multirow[t]{5}{*}{ SMR } & \multirow[t]{5}{*}{443} & & & \multirow[t]{5}{*}{ age, smoking habits } \\
\hline & & & & & $<50$ & & & 254 & $\begin{array}{c}0.00 \\
(0-12.50)\end{array}$ & \\
\hline & & & & & $50-990$ & & & 76 & $\begin{array}{c}0.00 \\
(0-47.05)\end{array}$ & \\
\hline & & & \multirow{2}{*}{$\begin{array}{l}\text { ICD9 188, } 189 \\
\text { ICDO } \\
\text { histology N/A }\end{array}$} & & $\geq 1,000$ & & & \multirow[t]{2}{*}{113} & \multirow{2}{*}{$\begin{array}{c}31.18 \\
(8.62-91.75)\end{array}$} & \\
\hline & & & & & $\begin{array}{l}\text { [Arsenic-polluted area. } \\
\text { Exposure to be between } \\
\text { 1955-59. All } 34 \text { wells in } \\
\text { the area were sampled } \\
\text { and arsenic concentration } \\
\text { ranged from non detect- } \\
\left.\text { able to } 3,000 \mu \mathrm{g} \cdot \mathrm{L}^{-1}\right) \text {.] }\end{array}$ & & & & & \\
\hline
\end{tabular}


Table 4 Summary results from cohort studies reporting on arsenic exposure and the risk of bladder cancer (Continued)

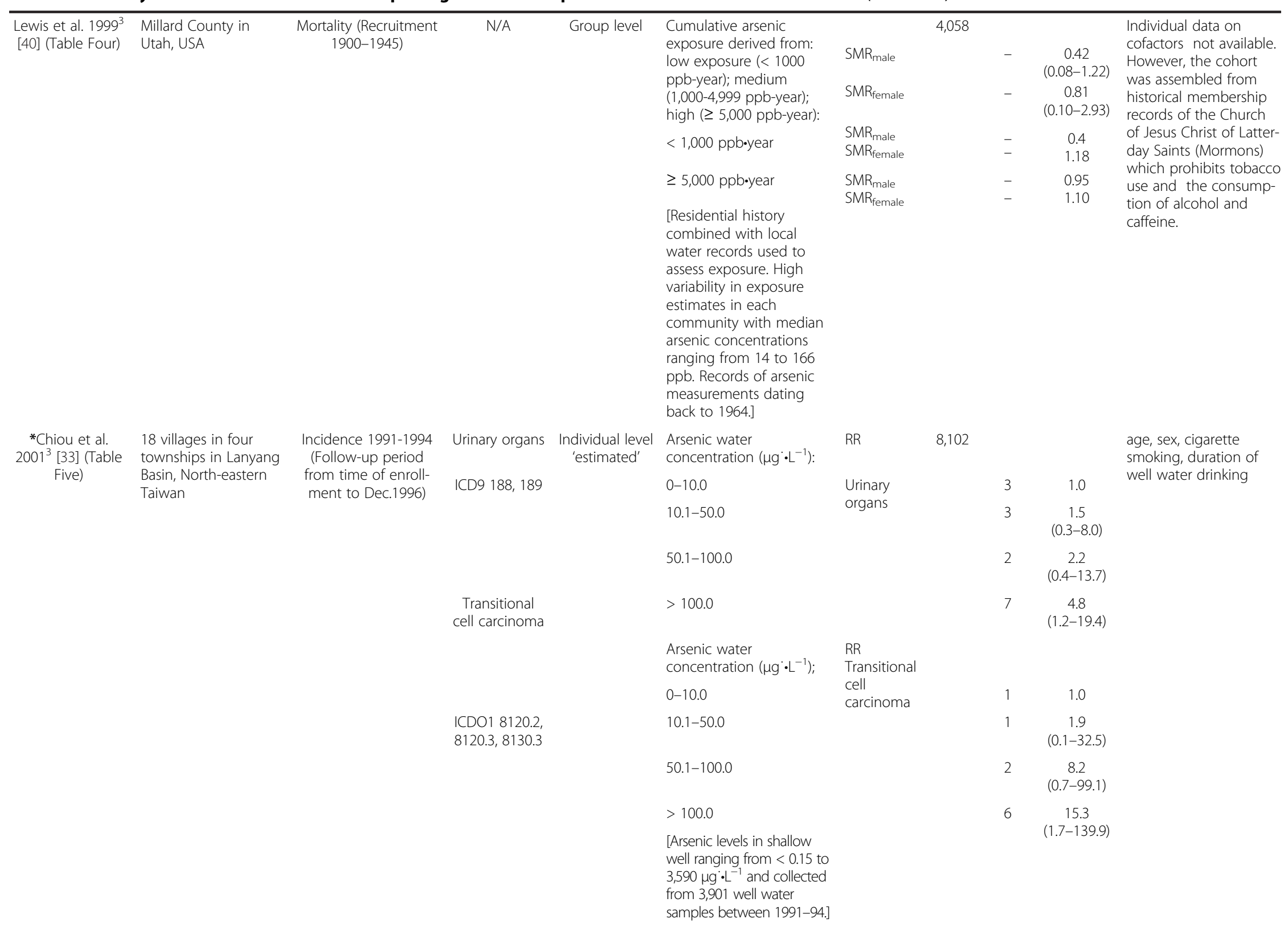


Table 4 Summary results from cohort studies reporting on arsenic exposure and the risk of bladder cancer (Continued)

\begin{tabular}{|c|c|c|c|c|c|c|c|c|c|c|}
\hline \multirow{3}{*}{$\begin{array}{c}\text { + Baastrup et al. } \\
2008 \text { [96] (Table } \\
\text { Three) }\end{array}$} & \multirow{3}{*}{$\begin{array}{l}23 \text { municipalities in } \\
\text { Copenhagen \& Asrhus } \\
\text { areas, Dannemark }\end{array}$} & \multirow{3}{*}{$\begin{array}{l}\text { Incidence 1993-1997 } \\
\text { (Follow-up from en- } \\
\text { rollment until date of } \\
\text { first cancer diagnosis, } \\
\text { emigration, death, or } \\
\text { Aug. 2003) }\end{array}$} & \multirow[t]{3}{*}{$\mathrm{N} / \mathrm{A}$} & \multirow[t]{3}{*}{$\begin{array}{l}\text { Individual level } \\
\text { 'estimated' }\end{array}$} & $\begin{array}{l}\text { Cumulated arsenic } \\
\text { exposure }(5 \mathrm{mg}) \text { : }\end{array}$ & IRR & \multirow[t]{3}{*}{56,378} & 214 & $\begin{array}{c}1.0 \\
(0.98-1.04)\end{array}$ & \multirow{3}{*}{$\begin{array}{l}\text { smoking status, } \\
\text { smoking duration, } \\
\text { smoking intensity, } \\
\text { education, occupation }\end{array}$} \\
\hline & & & & & $\begin{array}{l}\text { Time-weighted average } \\
\text { exposure }\left(\mu \mathrm{g} \cdot \mathrm{L}^{-1}\right) \text { : }\end{array}$ & IRR & & 214 & $\begin{array}{c}1.01 \\
(0.93-1.11)\end{array}$ & \\
\hline & & & & & $\begin{array}{l}\text { [Average arsenic exposure } \\
\text { from } 0.05 \text { to } 25.3 \mu \mathrm{g} \cdot \mathrm{L}^{-1} \\
\text { with mean of } 1.2 \mu \mathrm{\mu g} \cdot \mathrm{L}^{-1} \text {. } \\
\text { Average arsenic } \\
\text { concentrations obtained } \\
\text { from } 4,954 \text { samples from } \\
2,487 \text { water utilities } \\
\text { collected, 1987-2004, } \\
\text { with most samples dating } \\
\text { 2002-04. Residential } \\
\text { history } 1970-2003 \text {.] }\end{array}$ & & & & & \\
\hline \multirow{11}{*}{$\begin{array}{c}\text { *tHuang et al. } \\
2008 \text { [53] (Table } \\
\text { Two) }\end{array}$} & \multirow{11}{*}{$\begin{array}{l}3 \text { villages in Putai } \\
\text { Township, in BFD } \\
\text { endemic area of } \\
\text { southern Taiwan }\end{array}$} & \multirow{11}{*}{$\begin{array}{c}\text { Incidence } 1989 \\
\text { (Average follow-up } \\
\text { period of } 12 \text { years) }\end{array}$} & \multirow[t]{2}{*}{$\begin{array}{l}\text { Urothelial } \\
\text { carcinoma }\end{array}$} & \multirow[t]{11}{*}{$\begin{array}{l}\text { Individual level } \\
\text { 'estimated' }\end{array}$} & $\begin{array}{l}\text { Arsenic water } \\
\text { concentration }\left(\mu \mathrm{g} \cdot \mathrm{L}^{-1}\right) \text { : }\end{array}$ & RR & 1,078 & & & $\begin{array}{l}\text { age, sex, cigarette } \\
\text { smoking, education }\end{array}$ \\
\hline & & & & & $0-400$ & & & 1 & 1.0 & \\
\hline & & & \multirow{9}{*}{$\begin{array}{c}\text { ICDO3 M- } \\
\text { codes } 8120 / 3 \\
8230 / 3\end{array}$} & & $401-700$ & & & 14 & $\begin{array}{c}5.2 \\
(0.7-39.8)\end{array}$ & \\
\hline & & & & & $710-900$ & & & 9 & $\begin{array}{c}6.7 \\
(0.8-53.4)\end{array}$ & \\
\hline & & & & & $\geq 900$ & & & 7 & $\begin{array}{c}6.5 \\
(0.8-53.1)\end{array}$ & \\
\hline & & & & & $\begin{array}{l}\text { Cumulative arsenic } \\
\text { exposure }\left(\mathrm{mg} \cdot \mathrm{L}^{-1} \cdot \text { year): }\right.\end{array}$ & $\mathrm{RR}$ & & & & \\
\hline & & & & & 0 & & & 0 & - & \\
\hline & & & & & $0.1-11.9$ & & & 2 & 1.0 & \\
\hline & & & & & $12.0-19.9$ & & & 9 & $\begin{array}{c}4.6 \\
(1.0-21.8)\end{array}$ & \\
\hline & & & & & $\geq 20.0$ & & & 20 & $\begin{array}{c}7.9 \\
(17-379)\end{array}$ & \\
\hline & & & & & $\begin{array}{l}\text { [Period of arsenic water } \\
\text { samples collection not } \\
\text { reported. Participants } \\
\text { used artesian well water } \\
\text { more }>30 \text { years when } \\
\text { recruited. Information } \\
\text { from interview included } \\
\text { history of well-water con- } \\
\text { sumption, residential his- } \\
\text { tory, lifestyle factors]. }\end{array}$ & & & & & \\
\hline $\begin{array}{c}\text { *+Chen et al. } \\
2010^{5} \text { [60] (Tables } \\
\text { One, Two) }\end{array}$ & Taiwan & $\begin{array}{l}\text { Incidence 1991-1994 } \\
\text { (Average follow-up } \\
\text { period of } 11.6 \text { years) }\end{array}$ & $\begin{array}{l}\text { Urothelial } \\
\text { carcinoma }\end{array}$ & $\begin{array}{l}\text { Individual level } \\
\text { 'measured' }\end{array}$ & $\begin{array}{l}\text { Arsenic water } \\
\text { concentration }\left(\mu \mathrm{g} \cdot \mathrm{L}^{-1}\right) \text { : }\end{array}$ & $\mathrm{RR}$ & 8,086 & & & $\begin{array}{l}\text { age, sex, cigarette } \\
\text { smoking status, } \\
\text { education, alcohol }\end{array}$ \\
\hline
\end{tabular}


Table 4 Summary results from cohort studies reporting on arsenic exposure and the risk of bladder cancer (Continued)

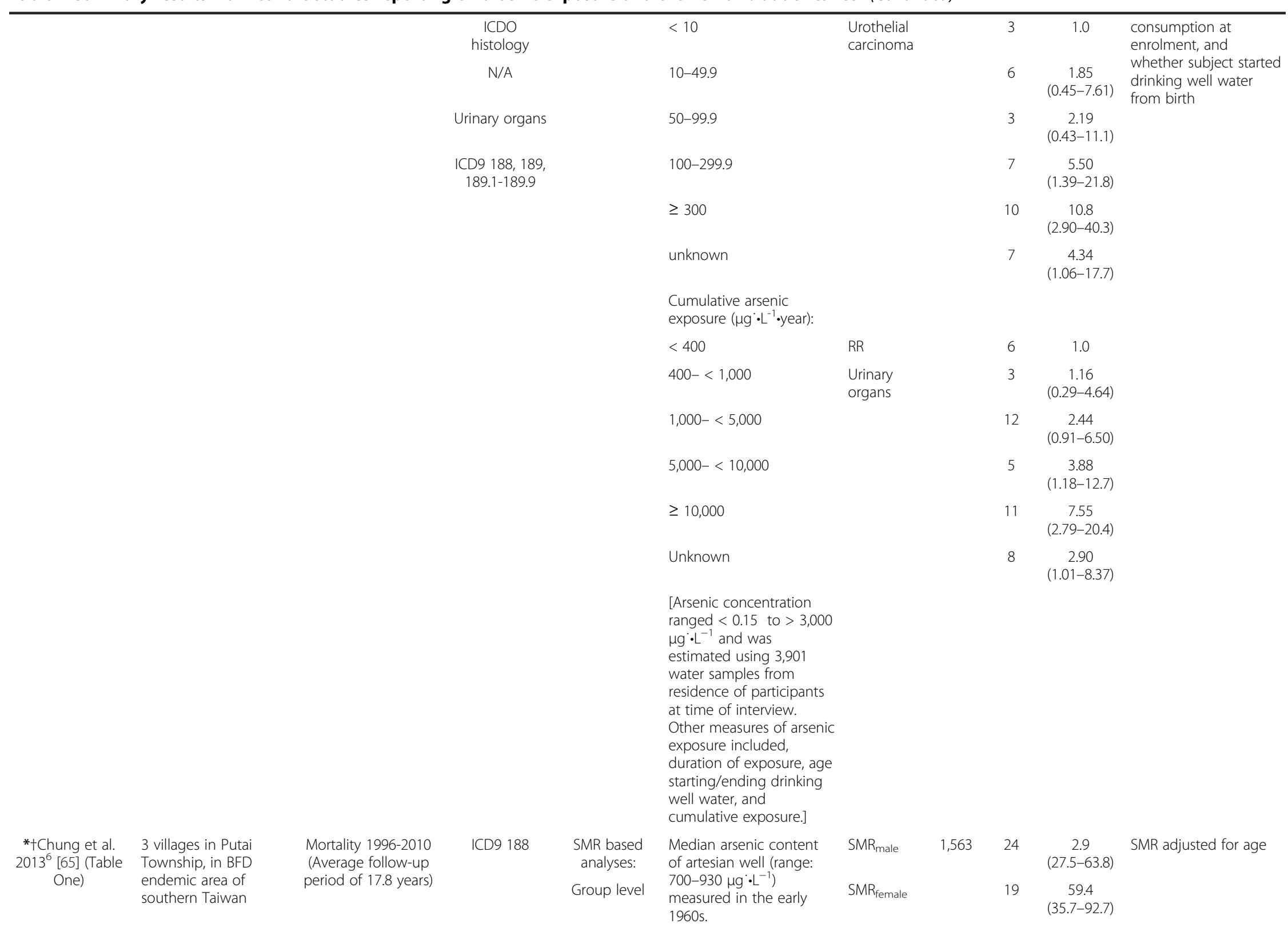




\begin{tabular}{|c|c|c|c|c|c|}
\hline & $\begin{array}{l}\text { [Used age-adjusted mor- } \\
\text { tality rate in Taiwan as } \\
\text { standard rates.] }\end{array}$ & & & & \\
\hline $\begin{array}{l}\text { HR based } \\
\text { analyses: } \\
\text { Individual level }\end{array}$ & $\begin{array}{l}\text { Average arsenic } \\
\text { concentration in artesian } \\
\text { well }\left(\mu \mathrm{g}^{\bullet} \cdot \mathrm{L}^{-1}\right) \text { : }\end{array}$ & $H R$ & & & \multirow[t]{5}{*}{$\begin{array}{l}\text { HR adjusted for age, } \\
\text { gender, education, } \\
\text { smoking habits }\end{array}$} \\
\hline & $<50$ & & 1 & 1.0 & \\
\hline & $50-710$ & & 15 & $\begin{array}{c}4.35 \\
(0.56-33.52)\end{array}$ & \\
\hline & $>710$ & & \multirow[t]{2}{*}{22} & \multirow{2}{*}{$\begin{array}{c}7.22 \\
(0.95-55.04)\end{array}$} & \\
\hline & $\begin{array}{l}\text { [Duration of drinking } \\
\text { artesian well water and } \\
\text { history of residential } \\
\text { address obtained from } \\
\text { questionnaires. Authors } \\
\text { found a significant } \\
\text { association with duration } \\
\text { of well water drinking.] }\end{array}$ & & & & \\
\hline
\end{tabular}

*Study included in meta-analyses.

tRecent study not included in the International Agency for Research on Cancer 2012 review (Monograph 100C [23]).

${ }^{1} \mathrm{ICD}=$ International Classification of Disease. ICD for cancer site abstracted which included bladder and urothelial/transitional cell carcinoma of the bladder or kidney. Transitional cell carcinoma of the renal pelvis often share the same etiology as bladder cancer, and as such, have been treated as bladder within the meta-analyses as recommended by IARC [23]. N/A = Not available. ${ }^{2}$ Cases $=$ number of persons exposed between 1955-1959.

${ }^{3} 95 \%$ Confidence intervals not available for data at low and high exposure.

${ }^{4}$ Results for transitional cell carcinoma were included in the meta-analysis.

${ }^{5}$ Results for urothelial carcinoma were included in the meta-analysis.

${ }^{6}$ Results from SMR were included in the meta-analyses. 
Table 5 Summary results from ecological studies reporting on arsenic exposure and kidney cancer

\begin{tabular}{|c|c|c|c|c|c|c|c|}
\hline $\begin{array}{l}\text { Study [reference] (Table } \\
\text { from original publication) }\end{array}$ & Study locale & Outcome & Exposure $^{1}$ [comments] & $I C D^{2}$ & Outcome measure & Cases & $\begin{array}{c}\text { Risk estimate } \\
(95 \% \mathrm{Cl})\end{array}$ \\
\hline \multirow[t]{2}{*}{$\begin{array}{c}\text { Chen et al. } 1985^{3} \text { [24] (Table } \\
\text { One) }\end{array}$} & $\begin{array}{l}84 \text { villages from } 4 \\
\text { neighbouring townships } \\
\text { on SW coast, Taiwan }\end{array}$ & Mortality 1968-82 & $\begin{array}{l}\text { Median arsenic content of artesian } \\
\text { well and (range): } 780 \mu \mathrm{\mu g} \cdot \mathrm{L}^{-1}(350- \\
\text { 1,140); in shallow well: } 40(0.0-300) \text {. } \\
\text { Period of samples collection not } \\
\text { reported. }\end{array}$ & ICD 189 & $S M R_{\text {male }}$ & 42 & $7.72(5.37-10.1)$ \\
\hline & & & $\begin{array}{l}\text { [Comparison of mortality rate in } \\
\text { Blackfoot disease (BFD) with those of } \\
\text { the general population.] }\end{array}$ & & $S M R_{\text {female }}$ & 62 & $11.2(8.38-14.0)$ \\
\hline \multirow[t]{10}{*}{$\begin{array}{c}\text { *Chen et al. 1988 [26] (Table } \\
\text { One) }\end{array}$} & $\begin{array}{l}\text { BFD endemic area, } \\
\text { Taiwan }\end{array}$ & Mortality $1973-86$ & $\begin{array}{l}\text { Arsenic well water concentration } \\
\left(\mu \mathrm{g}^{-} \mathrm{L}^{-1}\right) \text {. Period of samples } \\
\text { collection not reported. }\end{array}$ & ICD 189 & & & \\
\hline & & & General population & & $\mathrm{ASMR}_{\text {male }}$ & - & 1.1 \\
\hline & & & $<300$ & & & - & 5.4 \\
\hline & & & $300-590$ & & & - & 13.1 \\
\hline & & & $\geq 600$ & & & - & 21.6 \\
\hline & & & General population & & ASMR female & - & 0.9 \\
\hline & & & $<300$ & & & - & 3.6 \\
\hline & & & $300-590$ & & & - & 12.5 \\
\hline & & & $\geq 600$ & & & - & 33.3 \\
\hline & & & $\begin{array}{l}\text { [Comparison of mortality rate in BFD } \\
\text { with those of the general } \\
\text { population.] }\end{array}$ & & & & \\
\hline \multirow[t]{7}{*}{$\begin{array}{c}\text { *Wu et al. } 1989^{5} \text { [27] (Table } \\
\text { Three) }\end{array}$} & $\begin{array}{l}\text { BFD endemic area, } \\
\text { Taiwan (42 villages) }\end{array}$ & Mortality 1973-86 & $\begin{array}{l}\text { Arsenic well water concentration } \\
\left(\mu g \cdot L^{-1}\right) \text { based on well water } \\
\text { samples collected between 1964-66. }\end{array}$ & ICD8 189 & & & \\
\hline & & & $<300$ & & ASMR $_{\text {male }}$ & 9 & 8.42 \\
\hline & & & $300-590$ & & & 11 & 18.9 \\
\hline & & & $\geq 600$ & & & 6 & 25.3 \\
\hline & & & $<300$ & & $\mathrm{ASMR}_{\text {female }}$ & 4 & 3.42 \\
\hline & & & $300-590$ & & & 13 & 19.4 \\
\hline & & & $\geq 600$ & & & 16 & 58.0 \\
\hline $\begin{array}{c}\text { Chen and Wang } 1990^{6} \text { [28] } \\
\text { (Table Four) }\end{array}$ & $\begin{array}{l}314 \text { precincts \& } \\
\text { townships in Taiwan, }\end{array}$ & Mortality 1972-83 & $\begin{array}{l}\text { Average arsenic levels in water } \\
\text { samples of all } 314 \text { geographical }\end{array}$ & ICD 189 & & & \\
\hline
\end{tabular}


Table 5 Summary results from ecological studies reporting on arsenic exposure and kidney cancer (Continued)

\begin{tabular}{|c|c|c|c|c|c|c|c|}
\hline & $\begin{array}{l}\text { including } 4 \text { from BFD } \\
\text { endemic area }\end{array}$ & & $\begin{array}{l}\text { units. } 73.9 \% \text { had }<5 \% \text { of wells with } \\
>50 \mu \mathrm{g} \cdot \mathrm{L}^{-1} ; 14.7 \% \text { had } 5-14 \% \text {; } \\
11.5 \% \mathrm{had} \geq 15 \% \text {. Well water sam- } \\
\text { ples collected between } 1974-76 \text {. }\end{array}$ & & & & \\
\hline & & & All precincts \& townships & & $\mathrm{ASMR}_{\text {male }}$ & - & $1.1(0.2)$ \\
\hline & & & & & $\mathrm{ASMR}_{\text {female }}$ & - & $1.7(0.2)$ \\
\hline & & & Southwestern townships & & $\mathrm{ASMR}_{\text {male }}$ & - & $1.2(0.2)$ \\
\hline & & & & & ASMR female & - & $1.7(0.3)$ \\
\hline \multirow[t]{2}{*}{$\begin{array}{c}\text { Guo et al. 19977 [37] (Table } \\
\text { Two) }\end{array}$} & 243 townships in Taiwan & Incidence 1980-87 & $\begin{array}{l}\text { Arsenic well water concentration } \\
\text { ranging from }<50 \text { to }>640 \mu \mathrm{g} \cdot \mathrm{LL}^{-} \text {. }\end{array}$ & ICD 189.0, 189.1 & RDmale & - & $0.03(0.02)$ \\
\hline & & & $\begin{array}{l}\text { Estimate presented measured at }> \\
640 \mathrm{\mu g} \cdot \mathrm{L}^{-1} \text {. [Arsenic measurements } \\
\text { from a National survey of } 83,656 \\
\text { wells in } 243 \text { townships, collected } \\
\text { mostly between } 1974-76 . \text {.] }\end{array}$ & & RDfemale & - & $0.14(0.013)$ \\
\hline \multirow[t]{2}{*}{$\begin{array}{c}\text { Rivara et al.1997 [38] (Table } \\
\text { Four) }\end{array}$} & Chile & Mortality 1950-92 & $\begin{array}{l}\text { Annual average arsenic } \\
\text { concentration in drinking water for } \\
\text { Antofagasta (Region II of Chile) } \\
\text { ranging between } 40 \text { to } 860 \mu \mathrm{g} \cdot \mathrm{L}^{-1} \text {. } \\
\text { Data from historical records from } \\
\text { 1950-1992. }\end{array}$ & ICD 189 & RR & - & $3.8(3.1-4.7)$ \\
\hline & & & $\begin{array}{l}\text { [Comparison of mortality rate in } \\
\text { Region II (exposed) populations vs } \\
\text { Region VIII (control population.] }\end{array}$ & & & & \\
\hline \multirow[t]{2}{*}{ Smith et al. 1998 [39] } & Chile & Mortality 1989-93 & $\begin{array}{l}\text { Region II of Northern Chile with } \\
\text { population weighted average arsenic } \\
\text { concentration in drinking water up } \\
\text { to } 569 \mu g \cdot L^{-1} \text { compared with the } \\
\text { rest of Chile; exposure generally }<10 \\
\mu \mathrm{g} \cdot \mathrm{L}^{-1} \text {. }\end{array}$ & N/A & $\begin{array}{l}\text { SMR } \\
\text { male } \\
\text { SMR }_{\text {female }}\end{array}$ & $\begin{array}{l}39 \\
34\end{array}$ & $\begin{array}{l}1.6(1.1-2.1) \\
2.7(1.9-3.8)\end{array}$ \\
\hline & & & $\begin{array}{l}\text { [Arsenic measurements from 1950- } \\
\text { 94.] }\end{array}$ & & & & \\
\hline $\begin{array}{l}\text { Hinwood et al. } 1999 \text { [88] } \\
\text { (Table Two) }\end{array}$ & $\begin{array}{l}22 \text { areas in Victoria, } \\
\text { Australia }\end{array}$ & Incidence 1982-91 & $\begin{array}{l}\text { Median water arsenic concentration } \\
\text { ranging } 13 \mu \mathrm{g} \cdot \mathrm{L}^{-1} \text { to } 1,077 \mu \mathrm{g} \cdot \mathrm{L}^{-1} \text {. } \\
\text { [Selected areas were those where } \\
\text { samples with soil and/or water } \\
\text { arsenic concentration were generally } \\
\text { in excess of } 10 \mu \mathrm{\mu g} \cdot \mathrm{L}^{-1} \text {. Period for } \\
\text { samples collection is not available.] }\end{array}$ & ICD 189.0, 189.9 & SIR & 134 & $1.16(0.98-1.37)$ \\
\hline \multirow{3}{*}{$\begin{array}{c}\text { *Tsai et al. } 1999 \text { [41] (Tables } \\
\text { Two, Three) }\end{array}$} & \multirow{3}{*}{$\begin{array}{l}4 \text { townships from BFD } \\
\text { endemic area in SW } \\
\text { coast, Taiwan }\end{array}$} & \multirow[t]{3}{*}{ Mortality 1971-94 } & \multirow{2}{*}{$\begin{array}{l}\text { Median arsenic content of artesian } \\
\text { well: } 780 \mu \mathrm{g} \cdot \mathrm{L}^{-1} \text { (range: } 350-1,140 \text { ). }\end{array}$} & ICD 189 & $S_{M R}$ local-male & 94 & $6.76(5.46-8.27)$ \\
\hline & & & & & $\mathrm{SMR}_{\text {national-male }}$ & 94 & $6.80(5.49-8.32)$ \\
\hline & & & $\begin{array}{l}\text { Period of samples collection not } \\
\text { reported. Authors state that artesian }\end{array}$ & & SMR local-female & 128 & $8.89(7.42-10.6)$ \\
\hline
\end{tabular}




\section{*Study included in meta-analyses.}

tRecent study not included in the International Agency for Research on Cancer 2012 review (Monograph 100C [23]).

All ecological studies assessed arsenic exposure at the group-level.

$\mathrm{CD}=$ International Classification of Disease. N/A = not available.

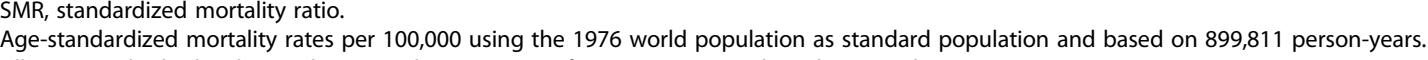

All age-standardardized mortality rates shown are significant at $p<0.001$ based on trend test.

Regression coefficient showing an increase in age-adjusted mortality per 100,000 persons-years for every 0.1 ppm increase in arsenic level, adjusting for indices of industrialization and urbanization. Standard errors

are in brackets. Kidney cancer was significantly correlated with average arsenic level in water.

$S M R_{\text {female_40 yearst }}$

SMR

$\mathrm{RD}$, rate difference (per 100,000 person-years) for one unit increase in the predictor and associated standard error for exposure $>640 \mu \mathrm{g} \cdot \mathrm{L}^{-1}(\mathrm{SE})$. 
Table 6 Summary results from cohort studies reporting on arsenic exposure and risk of kidney cancer

\begin{tabular}{|c|c|c|c|c|c|c|c|c|c|c|}
\hline $\begin{array}{l}\text { Study [reference] } \\
\text { (Table from } \\
\text { original } \\
\text { publication) }\end{array}$ & Study locale & Outcome & $\mathrm{ICD}^{1}$ & $\begin{array}{c}\text { Arsenic } \\
\text { exposure } \\
\text { assessment }\end{array}$ & Exposure [comments] & $\begin{array}{l}\text { Outcome } \\
\text { measure }\end{array}$ & $\begin{array}{l}\text { Cohort } \\
\text { size }\end{array}$ & Cases & $\begin{array}{c}\text { Risk } \\
\text { estimate } \\
(95 \% \mathrm{Cl})\end{array}$ & Covariates assessed \\
\hline $\begin{array}{l}\text { Chen et al. } 1988 \\
\text { [70] (Table Six) }\end{array}$ & $\begin{array}{l}4 \text { neighbouring } \\
\text { townships from } \\
\text { Blackfoot disease } \\
\text { (BFD) endemic area, } \\
\text { Taiwan }\end{array}$ & Morality 1968-83 & N/A & Group level & $\begin{array}{l}\text { Median arsenic content of artesian } \\
\text { well and (range): } 0.78 \mathrm{ppm}(0.35- \\
\text { 1.14); in shallow well: } 0.04(0.00-0.30) \text {. } \\
\text { General population used as } \\
\text { reference. } 95 \% \mathrm{Cl} \text { obtained from } \\
\text { IARC } 2012 \text { review [23]. }\end{array}$ & SMR & 871 & 3 & $\begin{array}{c}19.5 \\
(4.0-57.0)\end{array}$ & \\
\hline \multirow[t]{9}{*}{$\begin{array}{l}\text { Lewis et al. } 1999^{2} \\
\text { [40] (Table Four) }\end{array}$} & \multirow[t]{9}{*}{$\begin{array}{l}\text { Millard County in } \\
\text { Utah, USA }\end{array}$} & \multirow{9}{*}{$\begin{array}{l}\text { Mortality } \\
\text { (Recruitment } \\
\text { 1900-1945) }\end{array}$} & \multirow[t]{9}{*}{ N/A } & \multirow{9}{*}{ Group level } & \multirow{2}{*}{$\begin{array}{l}\text { Cumulative arsenic exposure derived } \\
\text { from: low exposure }(<1000 \mathrm{ppb}- \\
\text { year); medium ( } 1,000-4,999 \mathrm{ppb}- \\
\text { year); high ( } \geq 5,000 \text { ppb-year): }\end{array}$} & $S M R_{\text {male }}$ & \multirow[t]{9}{*}{4,058} & - & $\begin{array}{c}1.75 \\
(0.80-3.32)\end{array}$ & \multirow{9}{*}{$\begin{array}{l}\text { Individual data on cofactors not } \\
\text { available. However, the cohort } \\
\text { was assembled from historical } \\
\text { membership records of the } \\
\text { Church of Jesus Christ of Latter- } \\
\text { day Saints (Mormons) which pro- } \\
\text { hibits tobacco use and the con- } \\
\text { sumption of alcohol and caffeine. }\end{array}$} \\
\hline & & & & & & SMR female & & - & $\begin{array}{c}1.60 \\
(0.44-4.11)\end{array}$ & \\
\hline & & & & & \multirow[t]{2}{*}{$<1,000$ ppb•year } & $S M R_{\text {male }}$ & & - & 2.5 & \\
\hline & & & & & & $S M R_{\text {female }}$ & & - & 2.4 & \\
\hline & & & & & \multirow[t]{2}{*}{ 1,000 - 4,999 ppb·year } & $S M R_{\text {male }}$ & & - & 1.1 & \\
\hline & & & & & & $S M R_{\text {female }}$ & & - & 1.3 & \\
\hline & & & & & \multirow[t]{2}{*}{$\geq 5,000$ ppb•year } & $\mathrm{SMR}_{\text {male }}$ & & - & 1.4 & \\
\hline & & & & & & $S M R_{\text {female }}$ & & - & 1.1 & \\
\hline & & & & & $\begin{array}{l}\text { [Residential history combined with } \\
\text { local water records used to assess } \\
\text { exposure. High variability in exposure } \\
\text { estimates in each community with } \\
\text { median arsenic concentrations } \\
\text { ranging from } 14 \text { to } 166 \text { ppb. Records } \\
\text { of arsenic measurements dating } \\
\text { back to 1964.] }\end{array}$ & & & & & \\
\hline \multirow{3}{*}{$\begin{array}{c}\text { +Baastrup et al. } \\
2008 \text { [96] (Table } \\
\text { Three) }\end{array}$} & \multirow{3}{*}{$\begin{array}{l}23 \text { municipalities in } \\
\text { Copenhagen \& } \\
\text { Asrhus areas, } \\
\text { Dannemark }\end{array}$} & \multirow{3}{*}{$\begin{array}{l}\text { Incidence 1993- } \\
1997 \text { (Follow-up } \\
\text { from enrollment } \\
\text { until date of first } \\
\text { cancer diagnosis, } \\
\text { emigration, death, } \\
\text { or Aug. 2003) }\end{array}$} & \multirow[t]{3}{*}{ N/A } & \multirow{3}{*}{$\begin{array}{l}\text { Individual } \\
\text { level } \\
\text { 'estimated' }\end{array}$} & Cumulated arsenic exposure $(5 \mathrm{mg})$ : & IRR & \multirow[t]{3}{*}{56,378} & 53 & $\begin{array}{c}0.94 \\
(0.84-1.06)\end{array}$ & \multirow{3}{*}{$\begin{array}{l}\text { smoking status, smoking } \\
\text { duration, smoking intensity, } \\
\text { education, occupation }\end{array}$} \\
\hline & & & & & $\begin{array}{l}\text { Time-weighted average exposure } \\
\left(\mu \mathrm{g}^{\circ} \mathrm{L}^{-1}\right) \text { : }\end{array}$ & IRR & & 53 & $\begin{array}{c}0.89 \\
(0.65-1.21)\end{array}$ & \\
\hline & & & & & $\begin{array}{l}\text { [Average arsenic exposure from } 0.05 \\
\text { to } 25.3 \mu \mathrm{g} \cdot \mathrm{L}^{-1} \text {, with mean of } 1.2 \\
\mu \mathrm{g} \cdot \mathrm{L}^{-1} \text {. Average arsenic } \\
\text { concentrations obtained from } 4,954 \\
\text { samples from } 2,487 \text { water utilities } \\
\text { collected, } 1987-2004 \text {, with most } \\
\text { samples dating } 2002-04 \text {. Residential } \\
\text { history 1970-2003.] }\end{array}$ & & & & & \\
\hline
\end{tabular}

tRecent study not included in the International Agency for Research on Cancer 2012 review (Monograph 100C [23]).

${ }^{1} \mathrm{ICD}=$ International Classification of Disease. $\mathrm{N} / \mathrm{A}=$ not available.

${ }^{2} 95 \%$ Confidence intervals not available for data at low, medium and high exposure. 


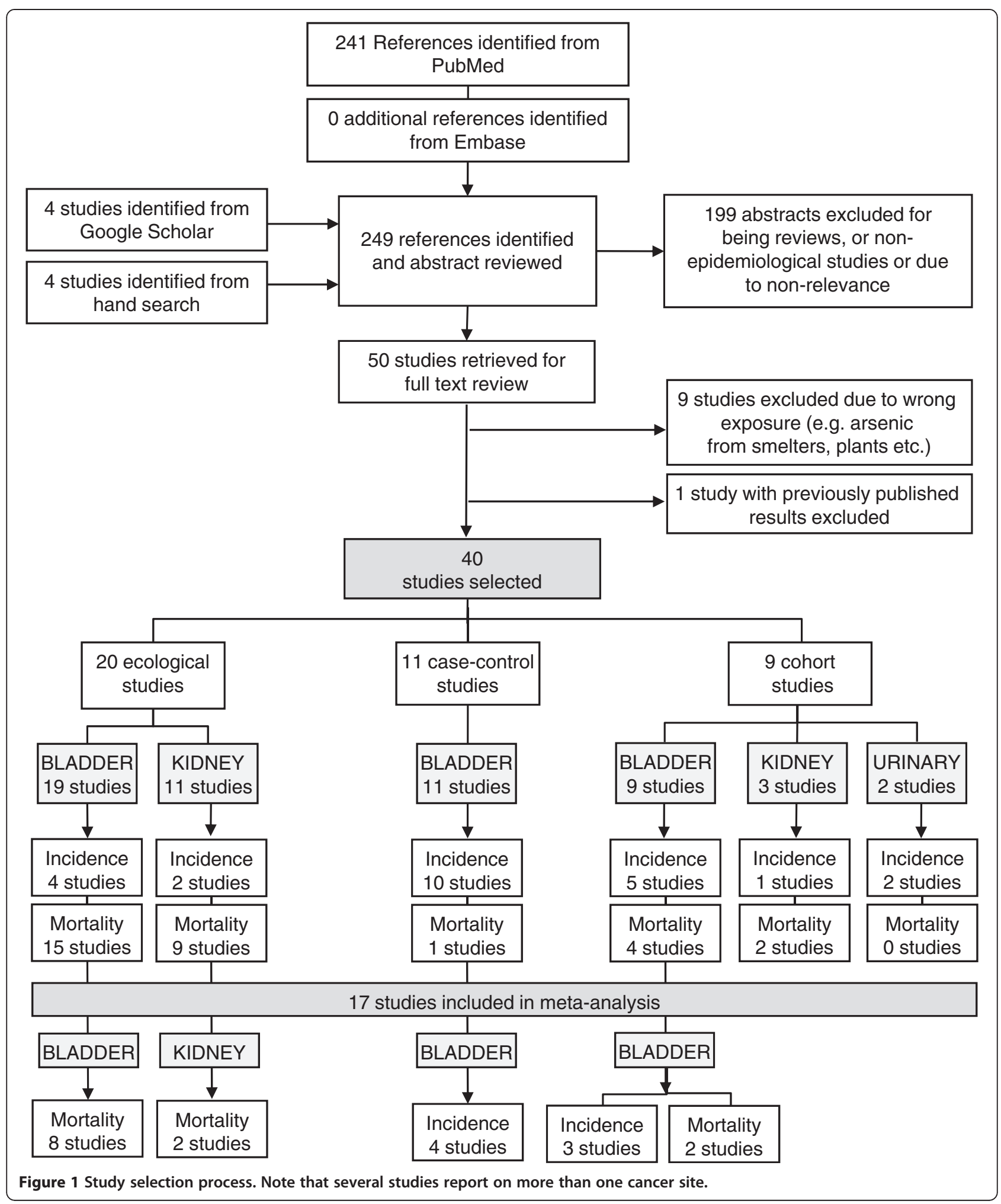

water (well or tap water), whereas all case-control and most cohort studies (7 of 9 studies) assessed As exposure using either a direct measure of As in tap/well water or body burden (e.g. urine or toenail As concentrations) or an individual level measure estimated from a range of metrics, including the reconstruction of past exposures based on residential history, knowledge of water source and duration of exposure to As contaminated well 


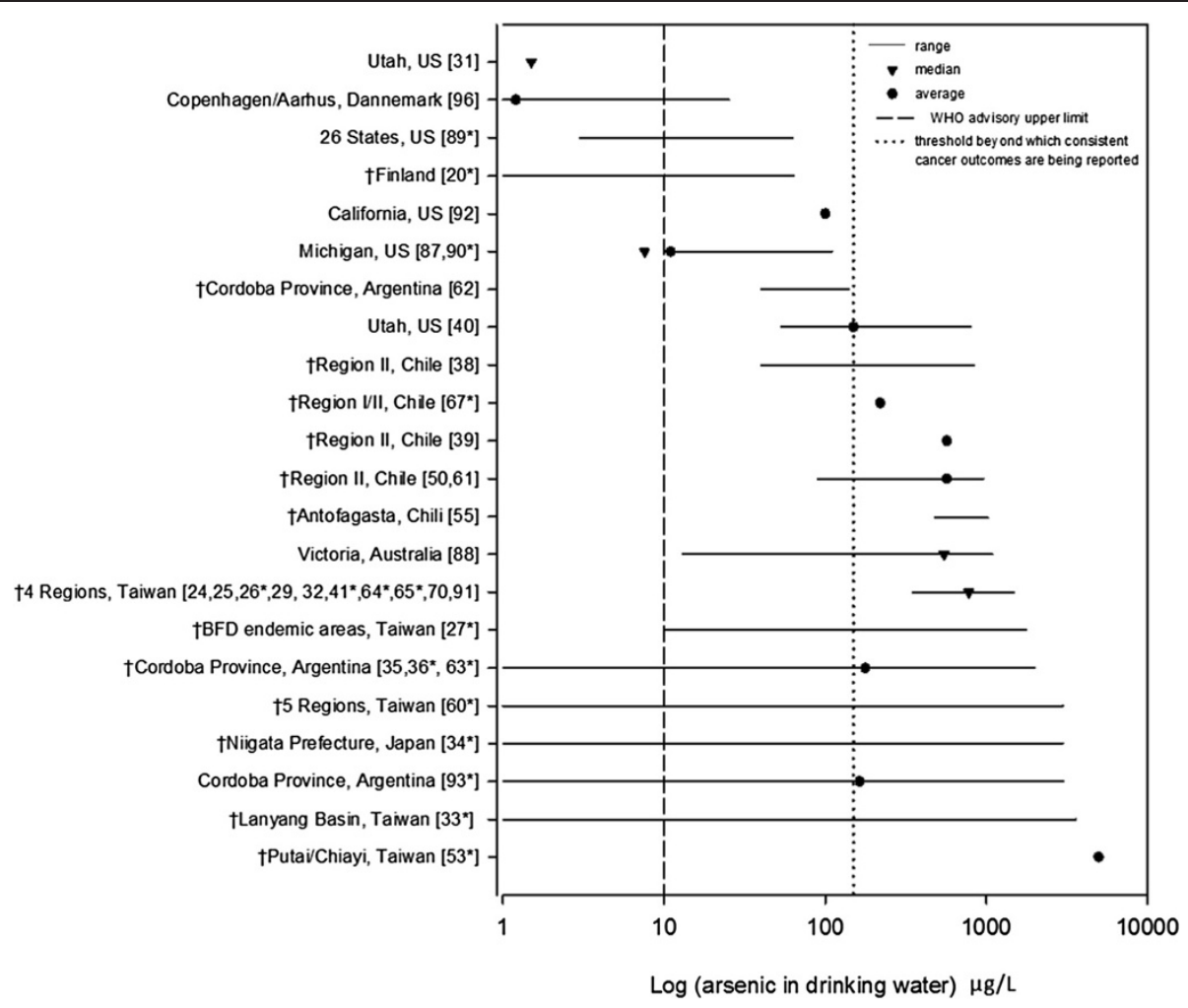

Figure 2 Arsenic concentrations from studies reporting on urinary tract cancers outcomes and arsenic exposure in drinking water. † indicates studies reporting significant associations and square brackets indicates citation number. Studies included in the meta-analysis are shown with an asterisk (*). Of the 40 studies reviewed, 3 used biomarkers to measure As exposure $[51,94,95]$ and 2 failed to provide a specific measure of As-concentration [28,37].

drinking water (see Table 2, 3, 4, 5, 6, As exposure assessment). Fifteen ecological studies and one cohort study stratified the analysis by gender (Tables $2,4,5,6)$. With the exception of one study [70], all case-control and cohort studies included in this review accounted for tobacco smoking and one ecological study used lung cancer mortality rates as surrogate to smoking [63].

\section{Arsenic exposure and bladder cancer Ecological studies}

Fifteen of the 20 ecological studies reviewed reported on bladder cancer mortality (Table 2). These studies provided consistent evidence for an increased risk of death from bladder cancer with exposure to As in drinking water. There were two exceptions, however, they focused only upon low exposures $(<60 \mu \mathrm{g} / \mathrm{L}$ As in water; $[89,90])$. Risk estimates amongst males and females were comparable, with the exception of those reported by Chen et al. [24] which showed a near doubling of risk in females on the southwest coast of Taiwan (Table 2). Chen [26] was also first to describe a dose-response relationship between well water As and rates of mortality from bladder cancer. In accordance with the three levels of As exposure examined (< 300; $300-590 ;>600 \mu \mathrm{g} / \mathrm{L} \mathrm{As})$, age-adjusted cancer mortality rates per 100,000 were as follows: 15.7 , 37.8, 89.1 per 100, 000 males and 16.7, 35.1, 91.5 per 100,000 females. While these findings profiled the highly exposed populations of Taiwan, increased mortality from bladder cancer due to As exposure in drinking water was also observed in Argentina [35,36,62,63] and Chile $[38,39,55]$. For example, compared to un-contaminated areas, males and females from the highly contaminated Region II of Chile, experienced mortality rates due to bladder cancer, 6.0 and 8.2 times greater, respectively [39]. Within the same region, Rivara et al. [38] reported on mortality rates of an order of magnitude higher (sex combined) relative to those observed in the rest of Chile. Findings from the 4 ecological studies reporting on bladder cancer incidence were generally consistent with those of studies based on mortality, providing evidence for an association between bladder cancer and exposure to As in drinking water. The exception was a study by Hinwood et al. [88] which was limited by low power and exposure misclassification.

\section{Case-control studies}

Ten of the 11 case-control studies reviewed reported on bladder cancer incidence [20,31,51,67,87,91-95]; one reported on mortality ([25]; Table 3 ). Four studies observed a 
significant As-related increase in bladder cancer incidence; one study observed an increased risk of death with increasing years of artesian well water consumption in Blackfoot disease endemic areas of Taiwan ([25]; Table 3). Two of these studies assessed As exposure from As in tap/well water, one from urine, one from cumulated exposure and one from years of artesian well water consumption. Three of the five studies reporting a significant association, also provided risk estimates by smoking status [20,31,51]. Two studies failed to find an effect among non-smokers [20,31]; one study reported a risk of about half the magnitude of that observed among smokers (never smokers: 4.4 [2.3 8.5] vs smokers: 8.2 [3.8 - 17.8]; Table 3) [51]. Regardless of the type of metric used to measure exposure (i.e. cumulative dose index, As in drinking water, body burden etc.), the risk of developing bladder cancer as a result of exposure to As, was consistently higher among smokers.

\section{Cohort studies}

Five of the 9 cohort studies reviewed reported on bladder cancer incidence $[32,33,53,60,96]$; four reported on mortality $(34,40,65,70]$; Table 4$)$. Seven of the 9 cohort studies showed an association between exposure to As contaminated drinking water and either bladder cancer incidence (4 studies, [32,33,53,60]) or mortality (3 studies, [34,65,70]). The work of both Chiou et al. [33] and Chen et al. [60] provided significant evidence for a dose-response relationship over a broad range of As exposure, from $<10 \mu \mathrm{g} / \mathrm{L}$ to $\geq$ $300 \mu \mathrm{g} / \mathrm{L}$. Chen et al. [60] report relative risk estimates for bladder cancer increasing from 1.9, 2.2, 5.5 and 10.8 for exposure to As ranging from < 10, 10 - 49.9, 50 - 99.9, 100 299.9 and $\geq 300 \mu \mathrm{g} / \mathrm{L}$, respectively. Consistent with these findings, Chiou et al. [33] report risks of similar magnitude, increasing from 1.9, 8.2, and 15.3 for exposure to As ranging from $10-50 \mu \mathrm{g} / \mathrm{L}, 50.1-100 \mu \mathrm{g} / \mathrm{L}$ and $>100 \mu \mathrm{g} / \mathrm{L}$, respectively. The largest cohort study involving 56,378 cases failed to provide evidence of an association [96]. However, average exposure ranged of 0.05 and $25.3 \mu \mathrm{g} / \mathrm{L}$ and mean exposure level was $1.2 \mu \mathrm{g} / \mathrm{L}$, with the authors indicating that only a small proportion of subjects were exposed to drinking-water containing As at $>2 \mu \mathrm{g} / \mathrm{L}$. Eight of the 9 cohort studies retained in this review adjusted for the effect of tobacco smoking [32-34,40,53,60,65,96].

\section{As exposure and kidney cancer Ecological studies}

Nine of the 20 ecological studies reviewed reported on kidney cancer mortality (Table 5). Eight of these studies provided evidence for an increased risk of death from kidney cancer with exposure to As in drinking water $[24,26-28,38,39,41,61]$; one study found no association [90]. At high levels of As exposure risk estimates were generally higher amongst females. Chen [26] was again, first to describe a dose-response relationship between well water As and rates of mortality from kidney cancer, reporting age-standardized rates increasing from: 5.4, 13.1, 21.6 per 100 , 000 males and $3.6,12.5,33.3$ per 100,000 females, with exposure to $<300,300-590$, and $>600 \mu \mathrm{g} / \mathrm{L}$ As, respectively (Table 5). Two ecological studies reported on kidney cancer incidence $[37,88]$ and one of these provided evidence for an association between kidney cancer and exposure to As in drinking water [37].

\section{Case-control studies}

None of the 11 case-control studies identified in this review reported on kidney cancer.

\section{Cohort studies}

One of the 9 cohort studies reported on kidney cancer incidence [96]; two reported on mortality [40,70] (Table 6). Of these 3 studies, one study showed a statistically significant increase in mortality with exposure to As contaminated drinking water [70]; the others reported a non significant increased risk in mortality [40] or incidence [96]. None of the cohort studies reviewed provided evidence for a dose-response relationship. Overall, as observed with ecological studies, the magnitude of the published risk estimates for kidney cancer was consistently lower than that observed for bladder or urinary organs cancer outcomes.

\section{Meta-analyses, Model I}

Analyses based on combined epidemiologic data showed an increase in the risk of developing bladder cancer or dying from bladder or kidney cancers with exposure to increasing levels of As in drinking water (Figure 3A-C). Combined bladder cancer SMRs ranged from $<1.0$ (As concentration mid-point $<10 \mu \mathrm{g} / \mathrm{L}$ ) to 38.8 (As concentration mid-point of $780 \mu \mathrm{g} / \mathrm{L}$; Figure 3A), showing a significant increase in risk at higher levels of exposure $\left(\mathrm{R}^{2}=0.96, \mathrm{p}<0.0001\right)$. Similarly, cancer mortality rates also significantly increased with increased well-water As (Figure 3B; $\mathrm{R}^{2}=0.92, \mathrm{p}<0.001$ ). However, the magnitude of the association was three times greater in those dying from bladder cancer relative to those dying from kidney cancer $(\mathrm{p}<0.0001)$. Bladder cancer mortality rates ranged from 15.7 (As mid-point of $150 \mu \mathrm{g} / \mathrm{L}$ ) to 91.5 per 100,000 persons (As mid-point of $870 \mu \mathrm{g} / \mathrm{L}$ ); kidney cancer mortality rates ranged from 5.4 (As mid-point of $150 \mu \mathrm{g} / \mathrm{L}$ ) to 58.0 per 100,000 persons (As mid-point of $870 \mu \mathrm{g} / \mathrm{L}$ ). Combined RRs for bladder cancer incidence studies, ranged from 1.0 (As mid-point of $5 \mu \mathrm{g} / \mathrm{L}$ ) to 15.3 (As mid-point of $1,845 \mu \mathrm{g} / \mathrm{L})$ and also indicated a statistically significant increase in risk with increasing well-water As (Figure 3C; $\left.\mathrm{R}^{2}=0.87, \mathrm{p}<0.0001\right)$. Predicted incidence risk of for bladder cancer increased 2.7 [1.2 - 4.1]; 4.2 [2.1 - 6.3] and; 5.8 [2.9 - 8.7], in those drinking water contaminated with $10 \mu \mathrm{g} / \mathrm{L} ; 50 \mu \mathrm{g} / \mathrm{L}$ and; $150 \mu \mathrm{g} / \mathrm{L}$ of As, respectively. 


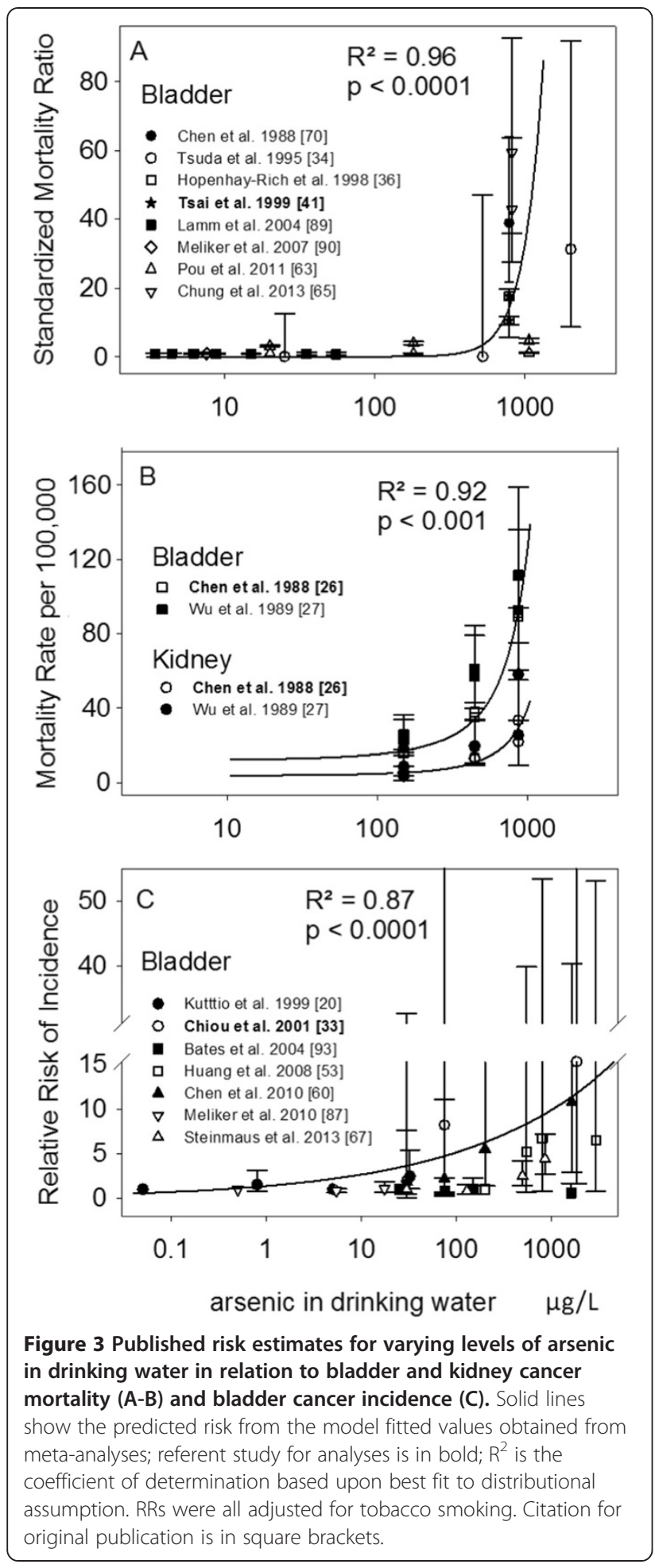

Meta-analyses, Model II

The robustness of the effect size at 10, 50 and $150 \mu \mathrm{g} / \mathrm{L}$ of As in drinking water for all three reported outcomes (mortality rates, SMR, RR) was assessed with Model II. The predicted risk derived from the bootstrapped randomizations (Figure 4A-D) confirms the non-linear increase in both bladder and kidney cancer mortality and in bladder cancer incidence with increasing levels of As in drinking water which was observed with Model I. However, the magnitude of the effect size for bladder cancer incidence (Figure 4D) was about $50 \%$ lower than those of Model I for exposure to 10,50 and $150 \mu \mathrm{g} / \mathrm{L}$ of As in drinking water: 1.4, 2.3 and 3.1(Model II) versus 2.7, 4.2 and 5.8 (Model I; Figure 4D). For bladder cancer mortality, the median SMR increased from 1.0 to 1.7 and 2.2 at 10,50 and $150 \mu \mathrm{g} / \mathrm{L}$, respectively. For both bladder and kidney cancers, mortality rates at $150 \mu \mathrm{g} / \mathrm{L}$ was about 30\% greater than those recorded at $10 \mu \mathrm{g} / \mathrm{L}$ (Figure $4 \mathrm{~A}-\mathrm{C}$ ). Although, these effect sizes were not statistically significant, they did follow a doseresponse relationship across all outcome measures. In addition, $51 \%$ and $65 \%$ of the probability density distribution in predicted SMRs and RRs, respectively, fells above 1.0 (no risk) at the lowest exposure benchmark of $10 \mu \mathrm{g} / \mathrm{L}$, with these proportions increasing to $74 \%$ and $83 \%$ for SMR and RR at levels of $50 \mu \mathrm{g} / \mathrm{L}$.

\section{Discussion}

\section{Summary of findings}

This review evaluated 40 studies reporting on the association between As in drinking water and urinary tract cancers. Evidence supporting an increased risk of developing, or dying from, bladder cancer as a result of exposure to As in drinking water was obtained from 28 studies from Taiwan, Chile, Argentina, Japan and Finland. Furthermore, evidence supporting an increased risk of developing, or dying from, kidney cancer due to As in drinking water was obtained from 10 studies from Taiwan and Chile. The risk associated with kidney cancer was consistently of lower magnitude than that reported for bladder cancer outcomes.

Twenty of the 40 studies reviewed were ecological by design, not accounting for potential confounders and with As exposure assigned using well water concentration from geographic or other grouped measurements, which could have resulted in the misclassification of exposure. However, the majority of these studies focused on highly exposed populations where the magnitude of the effects reported was so high that potential confounding or misclassification bias could not fully explain the associations.

Tabulated risk estimates from studies assessing exposure from As in well/tap drinking water, were generally measured within a limited range of As concentrations and varied across, and within regions, even in areas where similar concentrations of As had been measured. Differences in exposure (e.g. As species, timing and duration of exposure) [52] and population characteristics (e.g. genetic variations, lifestyle habits-smoking, diet etc.) have been suggested to contribute to differences in inter-individual susceptibility $[52,102,103]$. Thus, the methodological limitations of the 

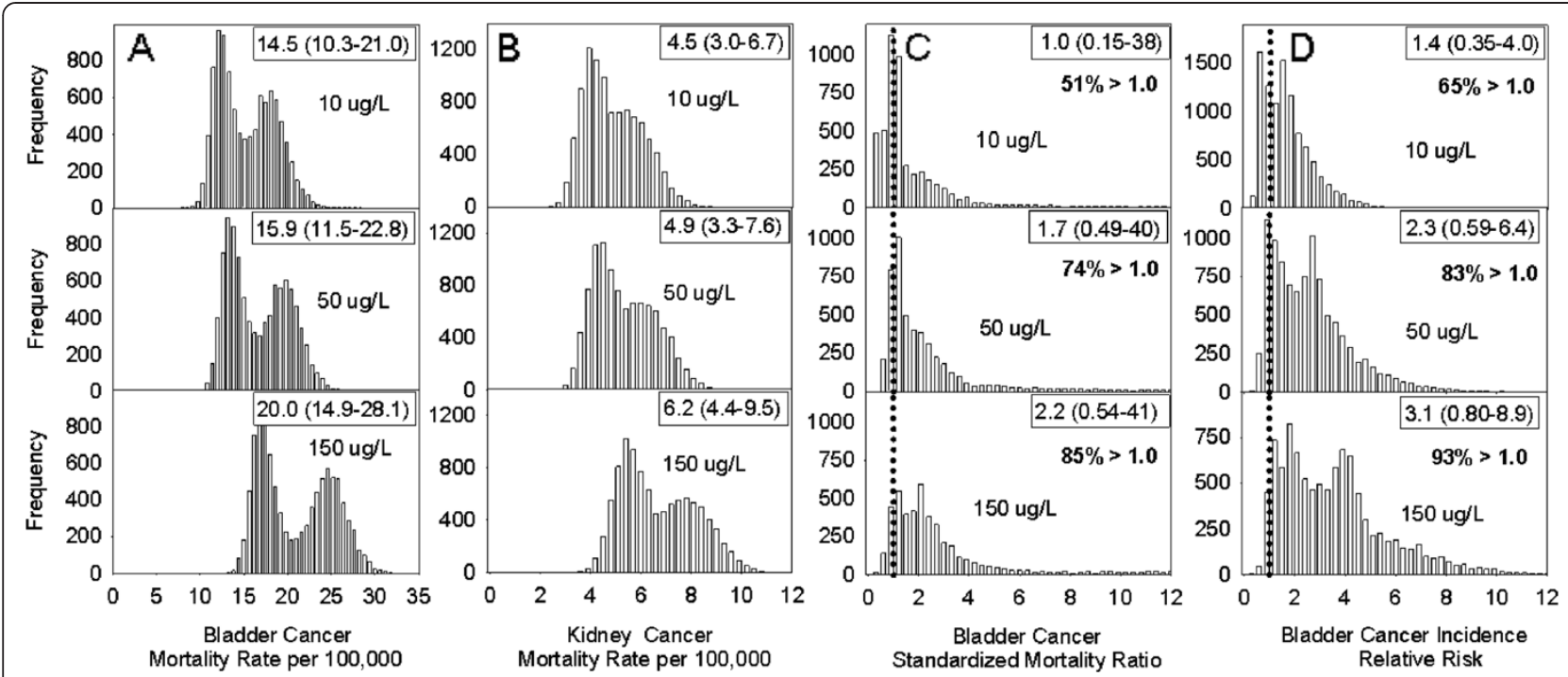

Figure 4 Distribution of predicted cancer risk estimates (A-B: mortality rates for bladder and kidney cancers; C: standardized mortality ratio for bladder cancer; D: incident relative risk for bladder cancer) at three levels of arsenic concentrations $(10,50$ and $150 \mu \mathrm{g} / \mathrm{L})$ in drinking water. Distributions were obtained from a bootstrap randomization of the fixed effects arsenic-risk models which were parameterized as a function of logged arsenic and the study from which the data were derived. A total of 10,000 randomizations were used.

studies reviewed, including study design, study quality (e.g. level of exposure assessment, lack of adjustment for potential confounders or effect modifiers such as age, sex, cigarette smoking, may have influenced the magnitude of the associations reported. For example, some case-control studies reporting on low exposure levels noted a significant association only among smokers [20,31] and of the cohort studies carried out in Taiwan, those adjusting for such covariates $[33,53,60]$ reported risk estimates three to fourfold lower than ecological studies that did not $[24,26]$.

\section{Meta-analysis of arsenic in drinking water and the risk of developing bladder or kidney cancers}

The analyses of combined risk estimates presented in this review allowed for the examination of the association between cancer outcomes (i.e. mortality and incidence) - independently, and As exposure over a broader and more continuous range of As concentrations. After adjusting for differences in unaccounted bias associated with each study, the results showed that exposure to increasing levels of As in drinking water was significantly associated with an increased risk of bladder and kidney cancer mortality and bladder cancer incidence, regardless of the measure of association employed (i.e. mortality rate, SMR, RR; Model I). Risk estimates obtained from fitted values from Model I showed that people exposed to drinking water contaminated with $10 \mu \mathrm{g} / \mathrm{L}$ of As had more than a twofold increased risk of developing bladder cancer (2.7 $[1.2-4.1])$; those exposed to $50 \mu \mathrm{g} / \mathrm{L}$ and $150 \mu \mathrm{g} / \mathrm{L}$ were expected of have a four- $(4.2[2.1-6.3])$ and six fold (5.8 [2.9 - 8.7) increase in risk, respectively- relative to the meta-analyses referent group (the general population of Taiwan). Sub-analyses focusing on low-level exposure ( $\leq$ $150 \mu \mathrm{g} / \mathrm{L}$ ) confirmed the trend, although the effect was slightly reduced at the $150 \mu \mathrm{g} / \mathrm{L}$ exposure level $(10 \mu \mathrm{g} / \mathrm{L}$, RR: 2.8 [1.3 - 4.3]; $50 \mu \mathrm{g} / \mathrm{L}, \mathrm{RR}: 3.7$ [1.7 - 5.7]; $150 \mu \mathrm{g} / \mathrm{L}$, RR: 4.5 [1.8 - 7.2]). A near six fold increase in bladder cancer risk was also observed by Chen et al. [60] in northeastern Taiwanese residents exposed to levels of As in drinking water ranging between 100-299.9 $\mu \mathrm{g} / \mathrm{L}$ (RR: 5.5 [1.4 - 22.0]). However, predicted risks for people exposed to 10 and $50 \mu \mathrm{g} / \mathrm{L}$ were about half of those obtained with Model I but comparable to those of Model II (Figure 4D; see also Chiou et al. [33] for a doubling of risk between $50-100 \mu \mathrm{g} / \mathrm{L}$ ). Of note, a recent review reporting on lowlevel As exposure in drinking water and bladder cancer did not support a significant association [56]. However, their findings were based on a meta-analytical approach that combined incidence and mortality outcomes, and studies using different metrics of exposure (e.g. As in toenails, well water, cumulated etc.), which possibly introduced statistical noise thereby attenuating the summary estimate (risk) towards the null. In this review, risk estimates derived from mortality were smaller than those of incidence data (Figure 4C-D). This possibly reflected patterns of prognosis [104], but perhaps more so, reduced statistical power due to misclassification as eight of the nine studies included in the meta-analyses of SMRs assessed exposure at the group-level, whereas all studies included in the analyses of the incidence data used individual-level measurements or estimations of As in drinking water. 
The precise magnitude of excess cancer risk associated with drinking water containing As has been difficult to establish, especially in populations exposed to moderate to low As-levels. A major issue relates to the misclassification of As exposure arising from uncertainties in assessing exposures during the disease-relevant exposure period, which, for As, may extend many decades prior to diagnosis. These uncertainties relate to population mobility, characterization of drinking water sources, assignment of water As concentrations to subjects over time, assessment of fluid intake rates, assessment of dietary As intake, a likely major contributor to exposure in areas of low As-levels [103,105], and difficulties in measuring actual levels of As in drinking water as opposed to relying on estimated levels [56]. Such uncertainties lead to bias which typically results in an underestimation of the true risk - a risk that can be small but still biologically significant.

These uncertainties also act to increase the variability in the distribution of both the measured (e.g. Figure 3) and consequently, the predicted (e.g. Figure 4) risks, weakening the statistical significance of the risk estimate. Studies using biomarkers of exposure offer perhaps a way to reduce such uncertainties that create exposure misclassification. However, rather than limiting the dialogue around As-related health effects to a significance level, perhaps more informative is the high probability that a large proportion of people may be at elevated risk of dying from (Figure 4C, 51\% probability) or being diagnosed with bladder cancer (Figure 4D, 65\% probability), even at exposure levels as low as $10 \mu \mathrm{g} / \mathrm{L}$. In this review, we estimate that with exposure to $50 \mu \mathrm{g} / \mathrm{L}$ of As in drinking water there is a $83 \%$ probability for an elevated risk of developing bladder cancer and a $74 \%$ probability of elevated mortality. (Figures 4C, 4D). Yet, hundreds of millions of people worldwide rely upon drinking water containing As at these concentrations and consider them to be safe $[3,69]$.

\section{Limitations and strengths}

This review has some limitations. First, the search strategy was limited to computerized databases which could preferentially include studies with statistically significant findings [106,107]. While this is a concern, we are confident that publication bias was possibly minimal as a third of the studies included in this review presented nonsignificant results. Second, the analyses of combined risk estimates were limited to studies providing specific point estimates of As in drinking water, the most common metric of exposure reported. This selection reduced the number of studies eligible for meta-analyses but minimized heterogeneity associated with other exposure metrics such as cumulative As exposure or As concentrations in toenails or urine; two measures linked to population/individual-dependent factors (e.g. years of exposure, cumulated volume of contaminated water ingested, metabolic capacity etc.). Third, analyses were performed independently for studies reporting on different outcomes (i.e. cancer incidence vs. cancer mortality) and different measures of association (i.e. mortality rate, SMR, RR). This stratified approach reduced the statistical power required to analyze the combined data by sex and/or smoking status; the latter being an important effect modifier in the cancer-As relationship. Studies supporting a higher risk among ever smoker are growing in number and so predicted risks presented in this review may be conservative for populations with a high proportion of ever smokers.

Nonetheless, this review has important strengths. First, its broad scope allowed for the inclusion of 30 years of publications and a wide range of exposure from which combined analyses could be performed. Second, the use of a sensitive search strategy ensured a high level of search completeness. Third, while the independent analyses of incidence and mortality outcomes was presented as a limitation in terms of statistical power, it likely minimized possible ascertainment bias and exposure misclassification issues. This is because mortality data are generally less precise than incidence data and the survival rate for bladder cancer is relatively high. In addition, if survival for bladder cancer patients is related to As exposure, then mortality studies could be at greater risk of being confounded compared to incidence studies [104]. Furthermore, exposure in mortality studies is often derived from aggregate data which are more prone to misclassification and bias. Finally, this review updates and complements previously published work, but also provides data which quantifies the risk of developing bladder cancer at varying levels of As exposure, including that observed at lower levels exposure.

\section{Conclusions}

Epidemiological studies provide extensive evidence in support of a causal association between exposure to higher levels of As concentrations in drinking water and the risk of developing or dying from bladder cancer, although the thresholds at which health effects develop remain uncertain at lower levels of As exposure in drinking water. Evidence in support of an increased risk of dying from kidney cancer with exposure to As is also accumulating, but studies reporting on incidence are lacking.

The results of the meta-analysis were consistent with the generally observed findings from the full body of literature reporting on bladder and kidney cancer outcomes and As-exposure. They also confirmed patterns of dose-responses within exposed populations and quantified the evidence for potential health effects at the lower end of the exposure curve where most uncertainties remain. This meta-analysis suggests that populations 
exposed to $150 \mu \mathrm{g} / \mathrm{L}$ As in drinking water may be increasing their risk of dying from bladder or kidney cancer by $30 \%$ relative to those exposed to $10 \mu \mathrm{g} / \mathrm{L}$. In addition, populations exposed to As concentrations as low as $10 \mu \mathrm{g} / \mathrm{L}$ in drinking water, (which corresponds to the WHO provisional guideline), may be doubling their risk of developing bladder cancer, or at the very least, increase it by about $40 \%$ compared to the unexposed populations included in the meta-analyses.

Thus, with the large number of people likely exposed to As in drinking water at the lower range of concentrations throughout the world, we suggest that the public health consequences of As in drinking water may be substantial. And as such, the current advisory limit for concentration of As in drinking water should be reviewed as well as policies on the promotion and support of household water arsenic remediation activities. Further studies focusing on populations exposed to low As concentrations with exposure measured at the individual level (e.g. biomarker studies), are required to confirm the observed health effect suggested in this review.

\section{Abbreviations}

WHO: World Health Organization; As: Arsenic; PubMed: Public/Publisher MEDLINE; BMI: Body mass index.

\section{Competing interests}

The authors declare that they have no competing interests.

\section{Authors' contributions}

NSJ conducted the literature search for this review, specified the inclusion and exclusion criteria, abstracted published data, modeled combined risk estimates, constructed tables and figures, drafted and revised the manuscript; LP and TD supervised the review, reviewed the article critically for important intellectual content and provided important assistance in the interpretation. PB provided intellectual content and statistical advice to carry the meta-analyses. All of the authors gave final approval.

\section{Acknowledgements}

We are grateful to the Canadian Cancer Society, the Nova Scotia Health Research Foundation and the Canadian Institute for Health Research for funding this project. We thank Ron Dewar from Cancer Care Nova Scotia for his invaluable guidance and support.

\section{Author details}

${ }^{1}$ Cancer Care Nova Scotia, Surveillance and Epidemiology Unit, Room 560 Bethune Building, 1276 South Street, Halifax B3H 2Y9, Nova Scotia, Canada. ${ }^{2}$ Interdisciplinary PhD program, Dalhousie University, 6299 South Street, Room 314, PO Box 15000, Halifax B3H 4R2, Nova Scotia, Canada.

${ }^{3}$ Department of Pediatrics and Population Cancer Research Program, Dalhousie University, 1494 Carlton Street, PO Box 15000, Halifax B3H 4R2, Nova Scotia, Canada. ${ }^{4}$ Population Studies and Surveillance, Cancer Care Ontario, 620 University Ave, Toronto M5G 2 L7, Ontario, Canada.

Received: 10 June 2013 Accepted: 5 March 2014

Published: 2 June 2014

\section{References}

1. Mandal BK, Suzuki KT: Arsenic round the world: a review. Talanta 2002, 58:201-235

2. Smedley PL, Kinniburgh DG: A review of the source, behaviour and distribution of arsenic in natural waters. Appl Geochem 2002, 17:517-568

3. IARC: Arsenic in drinking-water. Lyon: International Agency for Research on Cancer; 2004:267.
4. Singh N, Kumar D, Sahu AP: Arsenic in the environment: effects on human health and possible prevention. J Environ Biol 2007, 28:359-365.

5. Cantor KP, Lubin JH: Arsenic, internal cancers, and issues in inference from studies of low-level exposures in human populations. Toxicol Appl Pharmacol 2007, 222:252-257.

6. Mondal D, Banerjee M, Kundu M, Banerjee N, Bhattacharya U, Giri AK, Ganguli B, Sen Roy S, Polya DA: Comparison of drinking water, raw rice and cooking of rice as arsenic exposure routes in three contrasting areas of West Bengal, India. Environ Geochem Health 2010, 32:463-477.

7. Enterline PE, Day R, Marsh GM: Cancers related to exposure to arsenic at a copper smelter. Occup Environ Med 1995, 52:28-32.

8. Liu J, Zheng B, Aposhian HV, Zhou Y, Chen M-L, Zhang A, Waalkes MP. Chronic arsenic poisoning from burning high-arsenic-containing coal in Guizhou, China. Environ Health Perspect 2002, 110:119-122.

9. Silverman D, Deveda S, Moore L, Rothman N: Bladder Cancer. In Cancer Epidemiology and Prevention. New York: Oxford University Press; 2006:1101-1127.

10. World Health Organization: Arsenic, Fact sheet N³72, December 2012. http:// www.who.int/mediacentre/factsheets/fs372/en/.

11. Smith $\mathrm{AH}$, Lingas $\mathrm{EO}$, Rahman $\mathrm{M}$ : Contamination of drinking-water by arsenic in Bangladesh: a public health emergency. Bull World Health Organ 2000, 78:1093-1103.

12. Rahman MM, Chowdhury UK, Mukherjee SC, Mondal BK, Paul K, Lodh D, Biswas BK, Chanda CR, Basu GK, Saha KC, Roy S, Das R, Palit SK, Quamruzzaman Q, Chakraborti D: Chronic arsenic toxicity in Bangladesh and West Bengal, India-a review and commentary. J Toxicol Clin Toxicol 2001, 39:683-700.

13. Alam MGM, Allinson G, Stagnitti F, Tanaka A, Westbrooke M: Arsenic contamination in Bangladesh groundwater: a major environmental and social disaster. Int J Environ Health Res 2002, 12:235-253.

14. Lan C-C, Yu H-S, Ko Y-C: Chronic arsenic exposure and its adverse health effects in Taiwan: A paradigm for management of a global environmental problem. Kaohsiung J Med Sci 2011, 27:411-416.

15. Wang S, Mulligan CN: Natural attenuation processes for remediation of arsenic contaminated soils and groundwater. J Hazard Mater 2006, 138:459-470.

16. WHO: United Nations Synthesis report on arsenic in drinking water. Geneva, Switzerland: World Health Organization; 2003.

17. Rosas I, Belmont R, Armienta A, Baez A: Arsenic concentrations in water, soil, milk and forage in Comarca Lagunera, Mexico. Water Air Soil Pollut 1999, 112:133-149.

18. Börzsönyi M, Bereczky A, Rudnai P, Csanady M, Horvath A: Epidemiological studies on human subjects exposed to arsenic in drinking water in Southeast Hungary. Arch Toxicol 1992, 66:77-78.

19. Meranger JC: Arsenic in Nova Scotia groundwater. Sci Total Environ 1984 39:49.

20. Kurttio P, Pukkala E, Kahelin H, Auvinen A, Pekkanen J: Arsenic concentrations in well water and risk of bladder and kidney cancer in Finland. Environ Health Perspect 1999, 107:705-710.

21. Lubin $J H$, Beane Freeman LE, Cantor KP: Inorganic arsenic in drinking water an evolving public health concern. J Natl Cancer Inst 2007, 99:906-907.

22. Kumar A, Adak P, Gurian PL, Lockwood JR: Arsenic exposure in US public and domestic drinking water supplies: A comparative risk assessment. J Expo Sci Environ Epidemiol 2009, 20(3):245-254.

23. IARC: A Review of Human Carcinogens. C. Metals, Arsenic, Fibres and Dusts. Lyon: International Agency for Research on Cancer; 2012:41-93.

24. Chen C-J, Chuang Y-C, Lin T-M, Wu H-Y: Malignant neoplasms among residents of a Blackfoot disease-endemic area in Taiwan: High-Arsenic Artesian well water and cancers. Cancer Res 1985, 45:5895-5899.

25. Chen CJ, Chuang YC, You SL, Lin TM, Wu HY: A retrospective study on malignant neoplasms of bladder, lung and liver in blackfoot disease endemic area in Taiwan. Br J Cancer 1986, 53:399-405.

26. Chen CJ: Arsenic and cancers. Lancet 1988, 1:414-415.

27. Wu M-M, Kuo T-L, Hwang Y-H, Chen C-J: Dose-response relation between arsenic concentration in well water and mortality from cancers and vascular diseases. Am J Epidemiol 1989, 130:1123-1132.

28. Chen C-J, Wang C-J: Ecological correlation between Arsenic level in well water and age-adjusted mortality from Malignant Neoplasms. Cancer Res 1990, 50:5470-5474.

29. Chiang HS, Guo HR, Hong CL, Lin SM, Lee EF: The incidence of bladder cancer in the black foot disease endemic area in Taiwan. Br J Urol 1993, 71:274-278. 
30. Hertz-Picciotto I, Smith AH: Observations on the dose-response curve for arsenic exposure and lung cancer. Scand J Work Environ Health 1993, 19:217-226

31. Bates MN, Smith AH, Cantor KP: Case-control study of bladder cancer and arsenic in drinking water. Am J Epidemiol 1995, 141:523-530.

32. Chiou H-Y, Hsueh Y-M, Liaw K-F, Horng S-F, Chiang M-H, Pu Y-S, Shinn-Nan Lin J, Huang C-H, Chen C-J: Incidence of internal cancers and ingested inorganic arsenic: a seven-year follow-up study in Taiwan. Cancer Res 1995, 55:1296-1300.

33. Chiou HY, Chiou ST, Hsu YH, Chou YL, Tseng CH, Wei ML, Chen CJ: Incidence of transitional cell carcinoma and arsenic in drinking water: a follow-up study of 8,102 residents in an arseniasis-endemic area in northeastern Taiwan. Am J Epidemiol 2001, 153:411-418.

34. Tsuda T, Babazono A, Yamamoto E, Kurumatani N, Mino Y, Ogawa T, Kishi Y, Aoyama $\mathrm{H}$ : Ingested arsenic and internal cancer: a historical cohort study followed for 33 years. Am J Epidemiol 1995, 141:198-209.

35. Hopenhayn-Rich C, Biggs ML, Fuchs A, Bergoglio R, Tello EE, Nicolli H, Smith AH: Bladder cancer mortality associated with arsenic in drinking water in Argentina. Epidemiology 1996, 7:117-124.

36. Hopenhayn-Rich C, Biggs ML, Smith AH: Lung and kidney cancer mortality associated with arsenic in drinking water in Cordoba, Argentina. Int J Epidemiol 1998, 27:561-569.

37. Guo H-R, Chiang H-S, Hu H, Lipsitz SR, Monson RR: Arsenic in drinking water and incidence of urinary cancers. Epidemiology 1997, 8:545-550.

38. Rivara MIZ, Cebrian MG, Corey G, Hernandez MA, Romieu I: Cancer risk in an arsenic-contaminated area of Chile. Toxicol Ind Health 1997, 13:321-338.

39. Smith AH, Goycolea M, Haque R, Biggs ML: Marked increase in bladder and lung cancer mortality in a region of northern Chile Due to arsenic in drinking water. Am J Epidemiol 1998, 147:660-669.

40. Lewis DR, Southwick JW, Ouellet-Hellstrom R, Rench J, Calderon RL: Drinking water arsenic in Utah: A cohort mortality study. Environ Health Perspect 1999, 107:359-365.

41. Tsai SM, Wang TN, Ko YC: Mortality for certain diseases in areas with high levels of arsenic in drinking water. Arch Environ Health 1999, 54:186-193.

42. Ferreccio C, González Psych C, Milosavjlevic Stat V, Marshall Gredis G, Sancha AM: Lung cancer and arsenic exposure in drinking water: a case-control study in northern Chile. Cad Saude Publica 1998, 14(Suppl 3):193-198.

43. Morales $\mathrm{KH}$, Ryan L, Kuo TL, Wu MM, Chen CJ: Risk of internal cancers from arsenic in drinking water. Environ Health Perspect 2000, 108:655-661.

44. Yang M-H, Chen K-K, Yen C-C, Wang W-S, Chang Y-H, Huang WJ-S, Fan FS, Chiou T-J, Liu J-H, Chen P-M: Unusually high incidence of upper urinary tract urothelial carcinoma in Taiwan. Urology 2002, 59:681-687.

45. Khan MMH, Sakauchi F, Sonoda T, Washio M, Mori M: Magnitude of arsenic toxicity in tube-well drinking water in Bangladesh and its adverse effects on human health including cancer: evidence from a review of the literature. Asian Pac J Cancer Prev 2003, 4:7-14.

46. Chiu H-F, Ho S-C, Yang C-Y: Lung cancer mortality reduction after installation of tap-water supply system in an arseniasis-endemic area in Southwestern Taiwan. Lung Cancer 2004, 46:265-270.

47. Chen $\mathrm{Y}$, Ahsan $\mathrm{H}$ : Cancer burden from arsenic in drinking water in Bangladesh. Am J Public Health 2004, 94:741-744.

48. Hopenhayn C: Arsenic in drinking water: impact on human health. Elements 2006, 2:103-107.

49. Smith AH, Marshall G, Yuan Y, Ferreccio C, Liaw J, von Ehrenstein O, Steinmaus C, Bates MN, Selvin S: Increased mortality from lung cancer and bronchiectasis in young adults following exposure to arsenic in utero and early childhood. Environ Health Perspect 2006, 114(8)(8):1293-96.

50. Marshall G, Ferreccio C, Yuan Y, Bates MN, Steinmaus C, Selvin S, Liaw J, Smith AH: Fifty-year study of lung and bladder cancer mortality in Chile related to arsenic in drinking water. J Natl Cancer Inst 2007, 99:920-928.

51. Pu Y-S, Yang S-M, Huang Y-K, Chung C-J, Huang SK, Chiu AW-H, Yang M-H Chen C-J, Hsueh Y-M: Urinary arsenic profile affects the risk of urothelial carcinoma even at low arsenic exposure. Toxicol Appl Pharmacol 2007, 218:99-106

52. Celik I, Gallicchio L, Boyd K, Lam TK, Matanoski G, Tao X, Shiels M, Hammond E, Chen L, Robinson KA, Caulfield LE, Herman JG, Guallar E, Alberg AJ: Arsenic in drinking water and lung cancer: a systematic review. Environ Res 2008, 108:48-55.

53. Huang Y-K, Huang Y-L, Hsueh Y-M, Yang M-H, Wu M-M, Chen S-Y, Hsu L-I, Chen C-J: Arsenic exposure, urinary arsenic speciation, and the incidence of urothelial carcinoma: a twelve-year follow-up study. Cancer Causes Control 2008, 19:829-839.

54. Chung C-J, Huang C-J, Pu Y-S, Su C-T, Huang Y-K, Chen Y-T, Hsueh Y-M: Urinary 8-hydroxydeoxyguanosine and urothelial carcinoma risk in low arsenic exposure area. Toxicol Appl Pharmacol 2008, 226:14-21.

55. Fernández Ml, López JF, Vivaldi B, Coz F: Long-term impact of arsenic in drinking water on bladder cancer health care and mortality rates 20 years after end of exposure. J Urol 2012, 187:856-861.

56. Mink PJ, Alexander DD, Barraj LM, Kelsh MA, Tsuji JS: Low-level arsenic exposure in drinking water and bladder cancer: a review and meta-analysis. Regul Toxicol Pharmacol 2008, 52:299-310.

57. Heck JE, Nieves JW, Chen Y, Parvez F, Brandt-Rauf PW, Graziano JH, Slavkovich V, Howe GR, Ahsan H: Dietary intake of methionine, cysteine, and protein and urinary arsenic excretion in Bangladesh. Environ Health Perspect 2009, 117:99-104.

58. Sohel N, Persson LÅ, Rahman M, Streatfield PK, Yunus M, Ekström E-C, Vahter M: Arsenic in drinking water and adult mortality. Epidemiology 2009, 20:824-830.

59. Wade TJ, Xia Y, Wu K, Li Y, Ning Z, Le XC, Lu X, Feng Y, He X, Mumford L: Increased mortality associated with well-water arsenic exposure in Inner Mongolia, China. Int J Environ Res Public Health 2009, 6:1107-1123.

60. Chen C-L, Chiou H-Y, Hsu L-I, Hsueh Y-M, Wu M-M, Wang Y-H, Chen C-J: Arsenic in drinking water and risk of urinary tract cancer: a follow-up study from Northeastern Taiwan. Cancer Epidemiol Biomarkers Prev 2010, 19:101-110.

61. Yuan Y, Marshall G, Ferreccio C, Steinmaus C, Liaw J, Bates M, Smith AH: Kidney cancer mortality: fifty-year latency patterns related to arsenic exposure. Epidemiology 2010, 21:103-108.

62. Aballay LR, Diaz M del P, Francisca FM, Muñoz SE: Cancer incidence and pattern of arsenic concentration in drinking water wells in Córdoba, Argentina. Int J Environ Health Res 2012, 22(3):1-12

63. Pou SA, Osella AR, Diaz MDP: Bladder cancer mortality trends and patterns in Córdoba, Argentina (1986-2006). Cancer Causes Control 2011 22:407-415

64. Su C-C, Lu J-L, Tsai K-Y, Lian I-B: Reduction in arsenic intake from water has different impacts on lung cancer and bladder cancer in an arseniasis endemic area in Taiwan. Cancer Causes Control 2011, 22:101-108.

65. Chung C-J, Huang Y-L, Huang Y-K, Wu M-M, Chen S-Y, Hsueh Y-M, Chen C-J: Urinary arsenic profiles and the risks of cancer mortality: A population-based 20-year follow-up study in arseniasis-endemic areas in Taiwan. Environ Res 2013, 122:25-30

66. Leonardi G, Vahter M, Clemens F, Goessler W, Gurzau E, Hemminki K, Hough R, Koppova K, Kumar R, Rudnai P, Surdu S, Fletcher T: Inorganic arsenic and basal cell carcinoma in areas of Hungary, Romania, and Slovakia: a case-control study. Environ Health Perspect 2012, 120:721-726.

67. Steinmaus CM, Ferreccio C, Acevedo Romo J, Yuan Y, Cortes S, Marshall G, Moore LE, Balmes JR, Liaw J, Golden T, Smith AH: Drinking water arsenic in northern Chile: high cancer risks 40 years after exposure cessation. Cancer Epidemiol Biomarkers Prev 2013, 22(4):623-630.

68. Lamm SH, Robbins S, Zhou C, Lu J, Chen R, Feinleib M: Bladder/lung cancer mortality in Blackfoot-disease (BFD)-endemic area villages with low $(<150 \mu \mathrm{g} / \mathrm{L})$ well water arsenic levels - An exploration of the dose-response Poisson analysis. Regul Toxicol Pharmacol 2013, 65(1):147-156.

69. Christoforidou EP, Riza E, Kales SN, Hadjistavrou K, Stoltidi M, Kastania AN, Linos A: Bladder cancer and arsenic through drinking water: A systematic review of epidemiologic evidence. J Environ Sci Health A Tox Hazard Subst Environ Eng 2013, 48:1764-1775.

70. Chen CJ, Wu MM, Lee SS, Wang JD, Cheng SH, Wu HY: Atherogenicity and carcinogenicity of high-arsenic artesian well water. Multiple risk factors and related malignant neoplasms of blackfoot disease. Arteriosclerosis 1988, 8:452-460.

71. National Research Council: Critical Aspects of EPA's IRIS Assessment of Inorganic Arsenic: Interim Report. Washington, DC: The National Academies Press; 2014.

72. Karagas MR, Stukel TA, Tosteson TD: Assessment of cancer risk and environmental levels of arsenic in New Hampshire. Int J Hyg Environ Health 2002, 205:85-94

73. IARC: Monographs on the Evaluation of Carcinogenic Risks to Human: Drinking Water Disinfectants and Contaminants, including Arsenic. Lyon: International Agency for Research on Cancer; 2007.

74. Byrd DM, Roegner ML, Griffiths JC, Lamm SH, Grumski KS, Wilson R, Lai S: Carcinogenic risks of inorganic arsenic in perspective. Int Arch Occup Environ Health 1996, 68:484-494. 
75. Kitchin KT: Recent advances in arsenic carcinogenesis: modes of action, animal model systems, and methylated arsenic metabolites. Toxicol App/ Pharmacol 2001, 172:249-261.

76. Luster MI, Simeonova PP: Arsenic and urinary bladder cell proliferation Toxicol Appl Pharmacol 2004, 198:419-423

77. Cohen SM, Ohnishi T, Arnold LL, Le XC: Arsenic-induced bladder cancer in an animal model. Toxicol Appl Pharmacol 2007, 222:258-263.

78. Andrew AS, Mason RA, Kelsey KT, Schned AR, Marsit CJ, Nelson HH, Karagas MR: DNA repair genotype interacts with arsenic exposure to increase bladder cancer risk. Toxicol Lett 2009, 187:10-14.

79. Florea A-M, Büsselberg D: Arsenic trioxide in environmentally and clinically relevant concentrations interacts with calcium homeostasis and induces cell type specific cell death in tumor and non-tumor cells. Toxicology Letters 2008, 179:34-42.

80. Martinez VD, Becker-Santos DD, Vucic EA, Lam S, Lam WL: Induction of human squamous cell-type carcinomas by arsenic. Journal of Skin Cancer 2011, 2011:454157.

81. Ren X, McHale CM, Skibola CF, Smith AH, Smith MT, Zhang L: An emerging role for epigenetic dysregulation in arsenic toxicity and carcinogenesis. Environmental Health Perspectives 2011, 119(11):11-19.

82. Parkin DM: The global burden of urinary bladder cancer. Scand J Urol Nephrol Supp/ 2008, 218:12-20.

83. Zeegers MA, Kellen E, Buntinx F, Brandt P: The association between smoking, beverage consumption, diet and bladder cancer: a systematic literature review. World J Urol 2004, 21:392-401.

84. Lipworth L, Tarone RE, McLaughlin JK: The epidemiology of renal cell carcinoma. J Urol 2006, 176:2353-2358.

85. Burger M, Catto JWF, Dalbagni G, Grossman HB, Herr H, Karakiewicz P, Kassouf W, Kiemeney LA, la Vecchia C, Shariat S, Lotan Y: Epidemiology and risk factors of urothelial bladder cancer. Eur Urol 2012, 176.

86. Letašiová S, Medve'ová A, Šovčíková A, Dušinská M, Volkovová K, Mosoiu C, Bartonová A: Bladder cancer, a review of the environmental risk factors. Environ Health 2012, 11(Suppl 1):S11.

87. Meliker JR, Slotnick MJ, Avruskin GA, Schottenfeld D, Jacquez GM, Wilson ML, Goovaerts P, Franzblau A, Nriagu JO: Lifetime exposure to arsenic in drinking water and bladder cancer: a population-based case-control study in Michigan, USA. Cancer Causes Control 2010, 21(5):745-757.

88. Hinwood AL, Jolley DJ, Sim MR: Cancer incidence and high environmental arsenic concentrations in rural populations: results of an ecological study. Int J Environ Health Res 1999, 9:131-141.

89. Lamm SH, Engel A, Kruse MB, Feinleib M, Byrd DM, Lai S, Wilson R: Arsenic in drinking water and bladder cancer mortality in the United States: an analysis based on 133 U.S. counties and 30 years of observation. J Occup Environ Med 2004, 46:298-306.

90. Meliker JR, Slotnick MJ, Avruskin GA, Kaufmann A, Fedewa SA, Goovaerts P, Jacquez GJ, Nriagu JO: Individual lifetime exposure to inorganic arsenic using a space-time information system. Int Arch Occup Environ Health 2007, 80:184-197.

91. Chen Y-C, Su H-JJ, Guo Y-LL, Hsueh Y-M, Smith TJ, Ryan LM, Lee M-S, Christiani DC: Arsenic methylation and bladder cancer risk in Taiwan. Cancer Causes Control 2003, 14:303-310.

92. Steinmaus C, Yuan Y, Bates MN, Smith AH: Case-control study of bladder cancer and drinking water arsenic in the western United States. Am J Epidemiol 2003, 158:1193-1201.

93. Bates MN, Rey OA, Biggs ML, Hopenhayn C, Moore LE, Kalman D, Steinmaus C, Smith AH: Case-control study of bladder cancer and exposure to arsenic in Argentina. Am J Epidemio/ 2004, 159:381-389.

94. Karagas MR, Tosteson TD, Morris JS, Demidenko E, Mott LA, Heaney J, Schned A: Incidence of transitional cell carcinoma of the bladder and arsenic exposure in New Hampshire. Cancer Causes Control 2004, 15:465-472.

95. Michaud DS, Wright ME, Cantor KP, Taylor PR, Virtamo J, Albanes D: Arsenic concentrations in prediagnostic toenails and the risk of bladder cancer in a cohort study of male smokers. Am J Epidemiol 2004, 160:853-859.

96. Baastrup R, Sørensen M, Balstrøm T, Frederiksen $\mathrm{K}$, Larsen $\mathrm{CL}$, Tjønneland A Overvad K, Raaschou-Nielsen O: Arsenic in drinking-water and risk for cancer in Denmark. Environ Health Perspect 2008, 116:231-237.

97. Dobson AJ: An introduction to generalized linear models. 2nd edition. USA: CRC Press; 2010

98. Borenstein M, Hedges LV, Higgins JPT, Rothstein HR: Introduction to Meta-Analysis. UK: John Wiley \& Sons; 2011.
99. Efron B, Tibshirani RJ: An Introduction to the Bootstrap. New York: Chapman \& Hall; 1994

100. Tseng $\mathrm{C}-\mathrm{H}$ : A review on environmental factors regulating arsenic methylation in humans. Toxicol Appl Pharmacol 2009, 235:338-350.

101. R Development Core Team: R: A Language and Environment for Statistical Computing. Vienna, Austria: R Foundation for Statistical Compuing; 2011.

102. Genetic variations associated with interindividual sensitivity in the response to arsenic exposure. http://www.futuremedicine.com/doi/abs/ 10.2217/14622416.9.8.1113

103. EFSA Panel on contaminants in the Food Chain (CONTAM): Scientific Opinion on Arsenic in Food. EFSA Journal 2009, 7(10):199. Italy.

104. Kwong R, Karagas M, Kelsey K, Mason R, Tanyos S, Schned A, Marsit C, Andrew A: Arsenic exposure predicts bladder cancer survival in a US population. World J Urol 2010, 28:487-492.

105. Gilbert-Diamond D, Cottingham KL, Gruber JF, Punshon T, Sayarath V, Gandolfi AJ, Baker ER, Jackson BP, Folt CL, Karagas MR: Rice consumption contributes to arsenic exposure in US women. Proc Natl Acad Sci 2011, 108(51):20656-60

106. Conn VS, Isaramalai S, Rath S, Jantarakupt P, Wadhawan R, Dash Y: Beyond MEDLINE for literature searches. J Nurs Scholarsh 2003, 35:177-182.

107. Stroup DF, Berlin JA, Morton SC, Olkin I, Williamson GD, Rennie D, Moher D, Becker BJ, Sipe TA, Thacker SB, for the Meta-analysis Of Observational Studies in Epidemiology Group: Meta-analysis of observational studies in epidemiology: a proposal for reporting. JAMA 2000, 283:2008-2012.

doi:10.1186/1476-069X-13-44

Cite this article as: Saint-Jacques et al.: Arsenic in drinking water and urinary tract cancers: a systematic review of 30 years of epidemiological evidence. Environmental Health 2014 13:44.

\section{Submit your next manuscript to BioMed Central and take full advantage of:}

- Convenient online submission

- Thorough peer review

- No space constraints or color figure charges

- Immediate publication on acceptance

- Inclusion in PubMed, CAS, Scopus and Google Scholar

- Research which is freely available for redistribution 